\title{
A STRONGLY DEGENERATE PARABOLIC AGGREGATION EQUATION*
}

\author{
F. BETANCOURT ${ }^{\dagger}$, R. BÜRGER ${ }^{\ddagger}$, AND K.H. KARLSEN $^{\S}$
}

\begin{abstract}
This paper is concerned with a strongly degenerate convection-diffusion equation in one space dimension whose convective flux involves a nonlinear function of the total mass to one side of the given position. This equation can be understood as a model of aggregation of the individuals of a population with the solution representing their local density. The aggregation mechanism is balanced by a degenerate diffusion term describing the effect of dispersal. In the strongly degenerate case, solutions of the nonlocal problem are usually discontinuous and need to be defined as weak solutions. A finite difference scheme for the nonlocal problem is formulated and its convergence to the unique weak solution is proved. This scheme emerges from taking divided differences of a monotone scheme for the local PDE for the primitive. Some numerical examples illustrate the behaviour of solutions of the nonlocal problem, in particular the aggregation phenomenon.
\end{abstract}

Key words. Aggregation, strongly degenerate convection-diffusion equation, nonlocal flux, well-posedness, finite difference scheme.

AMS subject classifications. 35K65, 65N06, 92C99.

\section{Introduction}

1.1. Scope. This paper is related to the initial value problem for a strongly degenerate convection-diffusion equation of the form

$$
\begin{gathered}
u_{t}+\left(\Phi^{\prime}\left(\int_{-\infty}^{x} u(y, t) \mathrm{d} y\right) u(x, t)\right)_{x}=A(u)_{x x}, \quad x \in \mathbb{R}, \quad 0<t \leq T, \\
u(x, 0)=u_{0}(x) \geq 0, \quad x \in \mathbb{R}, \quad u_{0} \in\left(L^{1} \cap L^{\infty}\right)(\mathbb{R}),
\end{gathered}
$$

for the density $u=u(x, t) \geq 0$, where $A(u)$ is a diffusion function given by $A(u):=\int_{0}^{u} a(s) \mathrm{d} s$, where $a(u) \geq 0$ for $u \in \mathbb{R}$. The model (1.1), (1.2) was studied as a model of aggregation by a series of authors including Alt [1], Diaz, Nagai, and Shmarev [16], Nagai [30] and Nagai and Mimura [31, 32, 33], all of which assumed that $a(u)=0$ at at most isolated values of $u$. It is the purpose of this paper to study (1.1), (1.2) under the more general assumption that $a(u)=0$ on bounded $u$-intervals on which (1.1) reduces to a first-order conservation law with nonlocal flux. We assume that $A(s) \rightarrow \infty$ as $s \rightarrow \infty$.

The key observation made in previous work $[1,30,31,32,33]$ is that if all coefficients are sufficiently smooth, and $u(x, t)$ is an $L^{1}$ solution of the problem (1.1), (1.2), then the primitive defined by

$$
v(x, t):=\int_{-\infty}^{x} u(\xi, t) \mathrm{d} \xi, \quad t \in(0, T]
$$

\footnotetext{
*Received: June 22, 2010; accepted (in revised version): December 11, 2010. Communicated by Andrea Bertozzi.

†Departamento de Ingeniería Matemática, Facultad de Ciencias Físicas y Matemáticas, Universidad de Concepción, Casilla 160-C, Concepción, Chile (fbetanco@ing-mat.udec.cl).

${ }^{\ddagger} \mathrm{CI}^{2} \mathrm{MA}$ and Departamento de Ingeniería Matemática, Facultad de Ciencias Físicas y Matemáticas, Universidad de Concepción, Casilla 160-C, Concepción, Chile (rburger@ing-mat. udec.cl).

$\S$ Centre of Mathematics for Applications (CMA), University of Oslo, P.O. Box 1053, Blindern, N-0316 Oslo, Norway (kennethk@math.uio.no).
} 
is a solution of the local initial value problem

$$
\begin{gathered}
v_{t}+\Phi(v)_{x}=A\left(v_{x}\right)_{x}, \quad x \in \mathbb{R}, \quad t \in(0, T], \\
v(x, 0)=v_{0}(x), \quad x \in \mathbb{R}, \quad v_{0}(x):=\int_{-\infty}^{x} u_{0}(\xi) \mathrm{d} \xi .
\end{gathered}
$$

As a nonlinear but local PDE, (1.4) is more amenable to well-posedness and numerical analysis. In this work we use that the transformation to the local Equation (1.4) is also possible in the strongly degenerate case, in which solutions of (1.1) are usually discontinuous and need to be defined as weak solutions. We prove that any weak solution is also an entropy solution. This property allows us to use available $L^{1}$ stability and uniqueness results in the framework of entropy solutions.

The core, and essential novelty, of the paper is the formulation and convergence proof of a finite difference scheme for (1.1), (1.2) (in short, " $u$-scheme"). The scheme is based on a monotone difference scheme for the initial value problem (1.4), (1.5) (in short, " $v$-scheme") in the strongly degenerate case, which in turn is a special case of the schemes formulated and analyzed by Evje and Karlsen [19] for the more general doubly degenerate equation $v_{t}+\Phi(v)_{x}=B\left(A\left(v_{x}\right)\right)_{x}$. The $u$-scheme is obtained by taking finite differences of the numerical solution values generated by the $v$-scheme. The $v$-scheme is, in particular, monotonicity preserving, so the discrete approximations for $v$ are always monotonically increasing when the initial datum $v_{0}$ is, and therefore the $u$-scheme produces nonnegative solutions. Moreover, by modifications of standard compactness and Lax-Wendroff-type arguments it is proved that the numerical approximations generated by the $u$-scheme converge to the unique weak solution of (1.1), (1.2). An appealing feature is that the primitive (1.3) never needs to be calculated explicitly (except for the computation of $v_{0}$ ). Numerical examples illustrate the behaviour of solutions of (1.1), (1.2), and recorded error histories demonstrate the convergence of the $v$ - and $u$-schemes.

1.2. Assumptions. We assume that $u_{0}$ has compact support, and that there exists a constant $\mathcal{M}$ such that

$$
\operatorname{TV}\left(u_{0}\right)<\mathcal{M}
$$

We also need that $\Phi \in C^{2}(\mathbb{R})$, and that $\Phi$ has exactly one maximum:

$$
\exists v^{*}>0: \quad \Phi^{\prime}\left(v^{*}\right)=0, \quad \Phi^{\prime}(v)>0 \text { for } v<v^{*}, \quad \Phi^{\prime}(v)<0 \text { for } v>v^{*} .
$$

This assumption is introduced to facilitate some of the steps of our analysis; it is, however, not essential. In fact, in our convergence analysis of Section 4 we need to discuss the local behaviour of the numerical solution for $v$ close to where it includes the value $v^{*}$ since that value is critical in the definition of the numerical flux. If we employ a function $\Phi$ that has several separate extrema, then the locations of solution values including extrema are spatially well separated since the discrete analogue of $v_{x}$ is bounded, and the techniques of Section 4 can be extended to that case in a straightforward manner. We recall that the function $A$ is defined via

$$
A(u):=\int_{0}^{u} a(s) \mathrm{d} s, \quad \text { where } a(u) \geq 0 \text { for } u \in \mathbb{R} .
$$

The assumptions on $A$ are the following:

$$
A(s) \rightarrow \infty \quad \text { as } s \rightarrow \infty ; \quad \exists M_{a}>0: \quad a(s)<M_{a} \quad \text { for all } s \in \mathbb{R} .
$$


The first of these assumptions is fundamental in our analysis. If $A$ is bounded, then $v$ can become discontinuous and and we thus loose our $L^{\infty}$ bound on $u$. The second assumption, namely the boundedness of $a$, is mainly introduced to ensure that the scheme satisfies a well-defined CFL condition. An implicit version of the present scheme, which we do not consider herein, would possibly not require this assumption.

Our analysis is restricted to a finite final time $T$, since some of the constants appearing in the convergence analysis, which serves here as an existence proof, actually depend on $T$. The $L^{\infty}$ bound on $u$ is, however, independent of $T$.

1.3. Motivation. Equation (1.1), or some specific cases of it, were studied in a series of papers $[1,16,30,31,32,33]$, in all of which it is assumed that $a(u)=0$ at at most isolated values of $u$, so that it is always ensured that $A^{\prime}(u)>0$ for $u \geq 0$. The interpretation of (1.1) as a model of the aggregation of populations (e.g., of animals) can be illustrated as follows. Assume that $u(x, t)$ is the density of the population under study, and consider the equation

$$
u_{t}+\left(-k\left[\int_{-\infty}^{x} u(y, t) \mathrm{d} y-\int_{x}^{\infty} u(y, t) \mathrm{d} y\right] u\right)_{x}=A(u)_{x x}, \quad k>0 .
$$

Here, the convective term provides a mechanism that moves $u(x, t)$ to the right (respectively, to the left) if

$$
\int_{-\infty}^{x} u(y, t) \mathrm{d} y<\int_{x}^{\infty} u(y, t) \mathrm{d} y \quad(\text { respectively, } \ldots>\ldots) .
$$

In other words, an animal will move to the right (respectively, left) if the total population to its right is larger (respectively, smaller) than to its left. Now assume that the initial population is finite and define

$$
C_{0}:=\int_{\mathbb{R}} u_{0}(x) \mathrm{d} x
$$

It is then clear that (1.9) is an example of $(1.1)$ if $\Phi^{\prime}(v)=-k\left(2 v-C_{0}\right)$, i.e.,

$$
\Phi(v)=-k v\left(v-C_{0}\right)+\text { const. }
$$

The aggregation mechanism is balanced by nonlinear diffusion described by the term $A(u)_{x x}$, termed density-dependent dispersal in mathematical ecology. A novel feature addressed by the present analysis is a "threshold effect", i.e. dispersal only sets on when the density $u$ exceeds a critical value $u_{\mathrm{c}}>0$. The underlying idea is that the individuals, animals or humans, would react to variations of the local density only if that density exceeds a critical value. A similar "behavioristic" motivation of degenerate diffusion was advanced in the context of a traffic model, see [11,34]. This effect is considered in the present model since $A$ may degenerate on intervals. For example, for a constant $a_{0}>0$ we may consider

$$
a(u)=\left\{\begin{array}{ll}
0 & \text { for } u \leq u_{\mathrm{c}}, \\
a_{0} & \text { for } u>u_{\mathrm{c}},
\end{array} \quad \text { i.e., } \quad A(u)= \begin{cases}0 & \text { for } u \leq u_{\mathrm{c}}, \\
a_{0}\left(u-u_{\mathrm{c}}\right) & \text { for } u>u_{\mathrm{c}} .\end{cases}\right.
$$

To illustrate some of the consequences of the presence of a strongly degenerating diffusion term, and to compare our findings with the most recent results obtained 
for multi-dimensional aggregation equations, let us consider a strongly degenerating integrated diffusion coefficient $A(u)$ and the local degenerate parabolic PDE

$$
u_{t}+f(x, t, u)_{x}=A(u)_{x x}, \quad(x, t) \in \Pi_{T} ; \quad u(x, 0)=u_{0}(x), \quad x \in \mathbb{R},
$$

where $f$ should depend smoothly on $x$ and $u$. It is well known that even in the absence of a convective term $(f \equiv 0)$, i.e., for the problem

$$
u_{t}=A(u)_{x x}, \quad(x, t) \in \Pi_{T} ; \quad u(x, 0)=u_{0}(x), \quad x \in \mathbb{R},
$$

solutions of (1.13) may form discontinuities from smooth initial data in finite time due to the strong degeneracy of $A(u)$. The appearance of discontinuities motivates why solutions of strongly degenerate parabolic PDEs are studied as weak solutions. However, the appearance of discontinuities solely due to degenerate diffusion does not necessarily require the introduction of an entropy solution concept to ensure uniqueness. In fact, the uniqueness in $L^{1}$ of weak solutions of (1.14) is a classical result [10]. This result carries over to such cases of (1.13) that can be transformed to (1.14), for example the linear case $f(x, u)=\alpha u$, where $\alpha \in \mathbb{R}$ is a constant, or may possibly depend on $x$ and $t$ (in the latter case, restrictions on the choice of $\alpha(x)$ may apply).

This discussion motivates why we expect solutions of the problem (1.1), (1.2) to form discontinuities even from smooth initial data, so this problem should be studied in a suitably defined space of weak solutions. We may write (1.1) as

$$
u_{t}+\left(\Phi^{\prime}(v(x, t)) u\right)_{x}=A(u)_{x x} .
$$

In this work we demonstrate that for the present Equation (1.15) weak solutions are entropy solutions. The main importance of identifying weak solutions as entropy solutions lies in the easy access to stability and uniqueness results for entropy solutions (see [15, 22]) which can be applied to (1.1), (1.2), as will be done in Section 3.2.

1.4. Related work. More recently, aggregation equations of the form

$$
u_{t}+\nabla \cdot(u \nabla K * u)=\Delta A(u)
$$

have seen an enormous amount of interest, where the typical case is $A \equiv 0$. Here, $K$ denotes an interaction potential, and $K * u$ denotes spatial convolution. The nonlocal and diffusive terms account for long-range and short-range interactions, respectively, as is emphasized in [12]. The derivation of (1.16) from microscopic interacting particle systems and related models, and for particular choices of $K$ and $A$, is presented in $[4,9,12,28,29]$. Related models also include equations with fractional dissipation that cannot be cast in the form (1.16); see e.g. [25, 26].

The essential research problem associated with (1.16) (or variants of this equation) is the well-posedness of this equation together with bounded initial data $u(x, 0)=$ $u_{0}(x)$ for $x \in \mathbb{R}^{d}$, where $d$ denotes the number of space dimensions. While the shorttime existence of a unique smooth solution for smooth initial data is known in most situations, one wishes to determine criteria in terms of the functions $K$ and $A$ (or related diffusion terms), and possibly of $u_{0}$, that either ensure that smooth solutions exist globally in time, or that compel the solutions of (1.16) to blow up in finite time. This problem is analyzed in $[2,3,4,5,6,7,9,12,14,24,25,26,27]$ (this list is far from being complete). 
Here and in what follows, "blow-up" of a solution refers to $L^{\infty}$ norm blow-up (as opposed to the finite time loss of classical regularity generic to problems with degenerate diffusion). The occurrence of blow-up was analyzed in terms of the properties of $K$ for $A \equiv 0$ in $[4,5]$; if $K$ is radial, i.e., $K=K(|x|)$, then blow-up occurs if the Osgood condition for the characteristic ODEs is violated, as occurs e.g. for $K(x)=\exp (-|x|)$, while for a $C^{2}$ kernel this does not occur [4]. Li and Rodrigo [25, 26] consider this particular kernel and describe the circumstances under which blow-up occurs if the aggregation equation is equipped with fractional diffusion. Special cases of (1.16) have also been studied in the context of Patlak-Keller-Segel models, where $K$ is the fundamental solution to an elliptic PDE (see e.g. $[2,8]$ ).

We can write (1.1) as a one-dimensional version of (1.16) only in very special cases. However, and as was already pointed out in [31], (1.9) can be written as

$$
u_{t}+(u \tilde{K} * u)_{x}=A(u)_{x x},
$$

with the odd kernel $\tilde{K}(x)=-k \operatorname{sgn}(x)$. Equation (1.17), or equivalently, (1.1) with $\Phi$ given by (1.11), becomes a one-dimensional example of (1.16) if we observe that $\tilde{K} * u=K^{\prime} * u$, where $K^{\prime}$ denotes the derivative of $K$, if we choose the even kernel

$$
K(x)=-k|x|+C,
$$

where $C$ is a constant. We can write this as $K(x)=-\kappa(|x|)$ for $\kappa(r)=r-C$. Suppose that one uses this kernel in the multi-dimensional Equation (1.16). It is then straightforward to verify that in absence of dispersal $(A \equiv 0)$, the kernel (1.18) satisfies the integral condition for blow-up in finite time; see [4]. One result of our analysis is then that the condition (1.8) is sufficient to ensure that $L^{\infty}$ blow-up of solutions of (1.1) does not occur.

In fact, in the context of aggregation models that are based either on (1.1) or on the more recently studied Equation (1.16), the present work is the first that incorporates a strongly degenerate diffusion term, i.e., involves a function $A(u)$ that is flat on a $u$-interval of positive length. So far, diffusion terms that have been considered in (1.1) degenerate at at most isolated $u$-values. Nagai and Mimura [31] studied the Cauchy problem for Equation (1.1) under the assumptions $A(0)=0, A^{\prime}(u)>0$ being an odd function. The initial function for the Cauchy problem in [31] is assumed to be bounded, nonnegative and integrable. Nagai and Mimura [31] prove existence and uniqueness of a bounded and continuous solution to the initial value problem. In [32] the asymptotic behaviour of solutions to the same problem was studied for the specific choice

$$
A(u)=u^{m}, \quad m>1 .
$$

It seems that the analysis of (1.16) with degenerate diffusion has just started. Li and Zhang [27] study this equation in one space dimension for the diffusion function $A(u)=u^{3} / 3$, which degenerates at $u=0$ only. On the other hand, the numerical simulations presented herein show that under strongly degenerate diffusion, typical features of the aggregation phenomenon such as "clumped" solutions with very sharp edges [35] appear.

Let us briefly mention some of the recent results concerning (1.16). If diffusion is absent $(A \equiv 0),(1.16)$ becomes an inviscid nonlocal transport law, which are well known to be have better regularity properties than general quasi-linear conservation laws. In particular, Laurent [24] and Bertozzi and Laurent [5] show that if the initial 
condition is smooth, then solutions of (1.16) remain smooth for as long as the $L^{p}$ norms remains bounded. In particular, discontinuities can only occur if they were present in the initial data. Moreover, according to [27], the addition of nonlinear diffusion will cause higher regularity of weak solutions to be lost in finite time, i.e., the spatial gradient of the solution will experience $L^{\infty}$ blow-up in finite time. This contrasts with the expected solution behaviour of (1.1), (1.2) described in Section 1.3, namely that strong discontinuities form from smooth data.

Regarding uniqueness of weak solutions, it has been shown that in dimensions two and higher, entropy conditions are not required to ensure that weak solutions to (1.16) are unique. For the inviscid case, see Bertozzi and Brandman [3] or Bertozzi et al. [6]. For the case with diffusion, uniqueness is shown by Bertozzi and Slepčev in [7] and in more generality by Bedrossian et al. [2]. These results are consistent with ours.

1.5. Outline of the paper. The remainder of this paper is organized as follows. In Section 2 we state the definition of weak and entropy solutions of (1.1), (1.2). While it is standard to verify that any entropy solution is a weak solution, we are able to prove that for the present equation, any weak solution is an entropy solution. In Section 3.1 we state jump conditions that can be derived from the definition of weak solutions, and in Section 3.2 we prove the uniqueness of a weak solution, using that any weak solution is, in fact, an entropy solution. Section 4 presents a convergence analysis for the $u$-scheme. In Section 4.1, the schemes are described. Section 4.2 contains a series of lemmas stating uniform estimates on the numerical approximations generated by the $v$ - and the $u$-schemes, which allow to employ standard compactness arguments to deduce that both schemes converge to the unique weak solution. The final convergence result (Theorem 4.1) and its proof are presented in Section 4.3. This proof follows a standard Lax-Wendroff argument. Some numerical examples are presented in Section 5.

\section{Definition of a weak solution}

Definition 2.1. A measurable function $u$ is said to be a weak solution of the initial value problem (1.1), (1.2) if it satisfies the following conditions:

1. We have $u \in L^{\infty}\left(\Pi_{T}\right) \cap L^{\infty}(0, T ; B V(\mathbb{R}))$, and $A(u) \in L^{2}\left(0, T ; H^{1}(\mathbb{R})\right)$, where $\Pi_{T}:=\mathbb{R} \times(0, T)$.

2. The initial condition (1.2) is satisfied in the following sense:

$$
\lim _{t \downarrow 0} \int_{\mathbb{R}}\left|u(x, t)-u_{0}(x)\right| \mathrm{d} x=0 .
$$

3. If $v(x, t)$ is defined by (1.3), then the following equality is satisfied for all test functions $\phi \in C_{0}^{\infty}\left(\Pi_{T}\right)$ :

$$
\iint_{\Pi_{T}}\left\{u\left(\phi_{t}+\Phi^{\prime}(v) \phi_{x}\right)+A(u) \phi_{x x}\right\} \mathrm{d} x \mathrm{~d} t=0 .
$$

DeFinition 2.2. A measurable, nonnegative function $u$ is an entropy solution of (1.1), (1.2) if it satisfies items (1) and (2) of Definition 2.1 and if for all nonnegative test functions $\varphi \in C_{0}^{\infty}\left(\Pi_{T}\right)$, the following entropy inequality is satisfied:

$$
\begin{gathered}
\forall k \in \mathbb{R}: \iint_{\Pi_{T}}\left\{|u-k|\left(\varphi_{t}+\Phi^{\prime}(v) \varphi_{x}\right)-\operatorname{sgn}(u-k) u k \Phi^{\prime \prime}(v) \varphi\right. \\
\left.+|A(u)-A(k)| \varphi_{x x}\right\} \mathrm{d} x \mathrm{~d} t \geq 0 .
\end{gathered}
$$


It is straightforward to check that an entropy solution of the initial value problem (1.1), (1.2) is a weak solution.

Lemma 2.1. Assume that $u$ is an entropy solution of the initial value problem (1.1), (1.2) (cf. Definition 2.2). Then u is a weak solution (cf. Definition 2.1).

Proof. Choosing $k \geq\|u\|_{L^{\infty}\left(\Pi_{T}\right)}$ in (2.3) we obtain

$$
\iint_{\Pi_{T}}\left\{-(u-k)\left(\phi_{t}+\Phi^{\prime}(v) \phi_{x}\right)-A(u) \phi_{x x}\right\} \mathrm{d} x \mathrm{~d} t \geq-k \iint_{\Pi_{T}} u \Phi^{\prime \prime}(v) \phi \mathrm{d} x \mathrm{~d} t
$$

or equivalently,

$$
\iint_{\Pi_{T}}\left\{u\left(\phi_{t}+\Phi^{\prime}(v) \phi_{x}\right)+A(u) \phi_{x x}\right\} \mathrm{d} x \mathrm{~d} t \leq k \iint_{\Pi_{T}}\left\{\phi_{t}+\left(\Phi^{\prime}(v) \phi\right)_{x}\right\} \mathrm{d} x \mathrm{~d} t=0 .
$$

On the other hand, since we look for nonnegative solutions, it suffices to set $k=0$ in (2.3) to deduce that we always have

$$
\iint_{\Pi_{T}}\left\{u\left(\phi_{t}+\Phi^{\prime}(v) \phi_{x}\right)+A(u) \phi_{x x}\right\} \mathrm{d} x \mathrm{~d} t \geq 0
$$

Combining this with (2.4) we see that $u$ satisfies (2.2).

The following lemma states that conversely, any weak solution of the initial value problem (1.1), (1.2) is an entropy solution. Lemma 2.2 is inspired by Carrillo [13] and Kobayasi [23, Lemmas 3.1 and 3.3].

Lemma 2.2. Let $u$ be a weak solution of problem (1.1), (1.2). Then $u$ is also an entropy solution.

Proof. Let us define $\alpha(x, t):=\Phi^{\prime}(v(x, t))$. Then we recall that $u$ is a weak solution of (1.1) if for all test functions $\phi \in C_{0}^{\infty}\left(\Pi_{T}\right)$,

$$
\iint_{\Pi_{T}}\left\{u\left(\phi_{t}+\alpha(x, t) \phi_{x}\right)+A(u) \phi_{x x}\right\} \mathrm{d} x \mathrm{~d} t=0,
$$

or equivalently,

$$
\iint_{\Pi_{T}}\left\{u\left(\phi_{t}+\alpha(x, t) \phi_{x}\right)-A(u)_{x} \phi_{x}\right\} \mathrm{d} x \mathrm{~d} t=0 .
$$

In what follows we will utilize the functions defined by

$$
H_{0}(x):=\left\{\begin{array}{ll}
1 & \text { if } x>0, \\
0 & \text { if } x \leq 0,
\end{array} \quad H_{1}(x):=\left\{\begin{array}{ll}
1 & \text { if } x \geq 0, \\
0 & \text { if } x<0,
\end{array} \quad H_{\varepsilon}(x):= \begin{cases}1 & \text { if } x>\varepsilon, \\
x / \varepsilon & \text { if } x \in[0, \varepsilon], \\
0 & \text { if } x<0\end{cases}\right.\right.
$$

and the multi-valued function (see [13, 23])

$$
H(x):= \begin{cases}1 & \text { if } x>0 \\ {[0,1]} & \text { if } x=0 \\ 0 & \text { if } x<0 .\end{cases}
$$


To simplify the argument, let us concentrate on the case of a single $u$ interval $[m, M]$ of degeneracy, assuming that $A^{\prime}(s)=0$ for $s \in[m, M]$ and $A^{\prime}(s)>$ 0 for $s \notin[m, M]$, where $0 \leq m, M<\infty$. Now let us use as a test function $\phi(x, t)=H_{\varepsilon}(A(u)-A(k)) \varphi(x, t)$ with $k \notin[m, M]$, where $\varphi$ is an admissible test function. Following the proof of Lemma 2.4 in [22] we find

$$
\begin{aligned}
\iint_{\Pi_{T}}\left\{|u-k|^{+} \varphi_{t}\right. & +H_{0}(u-k)\left(\alpha(x, t)(u-k)-A(u)_{x}\right) \varphi_{x} \\
- & \left.H_{0}(u-k) \alpha_{x}(x, t) k \varphi\right\} \mathrm{d} x \mathrm{~d} t \geq 0 \quad \text { for } k \notin[m, M],
\end{aligned}
$$

where $|z|^{+}:=H_{0}(z) z$. Since $M<\infty$ we can construct a sequence $\left\{s_{n}\right\}_{n \in \mathbb{N}}$ such that $s_{n}>M, s_{n} \rightarrow M$, and $H_{0}\left(u-s_{n}\right) \rightarrow H_{0}(u-M)$ as $n \rightarrow \infty$. Setting $k=s_{n}$ in (2.6) and sending $n \rightarrow \infty$, we get

$$
\begin{gathered}
\iint_{\Pi_{T}}\left\{|u-M|^{+} \varphi_{t}+\alpha(x, t)|u-M|^{+} \varphi_{x}-H_{0}(u-M) A(u)_{x} \varphi_{x}\right. \\
\left.-H_{0}(u-M) \alpha_{x}(x, t) M \varphi\right\} \mathrm{d} x \mathrm{~d} t \geq 0 .
\end{gathered}
$$

Similarly, we may construct a sequence $\left\{s_{n}\right\}_{n \in \mathbb{N}}$ such that $s_{n}<m, s_{n} \rightarrow m$ and $H_{0}\left(u-s_{n}\right) \rightarrow H_{1}(u-m)$ as $n \rightarrow \infty$. Setting $k=s_{n}$ in (2.6) and sending $n \rightarrow \infty$ yields

$$
\begin{gathered}
\iint_{\Pi_{T}}\left\{|u-m|^{+} \varphi_{t}+\alpha(x, t)|u-m|^{+} \varphi_{x}-H_{1}(u-m) A(u)_{x} \varphi_{x}\right. \\
\left.-H_{1}(u-m) \alpha_{x}(x, t) m \varphi\right\} \mathrm{d} x \mathrm{~d} t \geq 0 .
\end{gathered}
$$

Now we take in the entropy inequality (2.8) a test function $\varphi(x, t)=\xi(x, t) \zeta(x, t)$, where $\zeta$ is a smooth function such that $0 \leq \zeta \leq 1$ and $\xi$ is an admissible test function, and in (2.7) we use $\varphi(x, t)=\xi(x, t)(1-\zeta(x, t))$. Adding both resulting expressions we obtain the inequality $I_{1}+I_{2}+I_{3}+I_{4} \geq 0$ with the following terms, where we drop the argument $(x, t)$ wherever convenient:

$$
\begin{aligned}
I_{1}:= & \iint_{\Pi_{T}}\left\{\left(|u-m|^{+}-|u-M|^{+}\right)(\xi \zeta)_{t}+|u-M|^{+} \xi_{t}\right\} \mathrm{d} x \mathrm{~d} t \\
I_{2}:= & \iint_{\Pi_{T}}\left\{\alpha\left(|u-m|^{+}-|u-M|^{+}\right)(\xi \zeta)_{x}+\alpha|u-M|^{+} \xi_{x}\right\} \mathrm{d} x \mathrm{~d} t, \\
I_{3}:= & \iint_{\Pi_{T}}\left\{\alpha_{x}\left(H_{0}(u-M) M-H_{1}(u-m) m\right) \xi \zeta-\alpha_{x} H_{0}(u-M) M \xi\right\} \mathrm{d} x \mathrm{~d} t, \\
I_{4}:= & -\iint_{\Pi_{T}}\left(H_{1}(u-m)-H_{0}(u-M)\right) A(u)_{x}(\xi \zeta)_{x} \mathrm{~d} x \mathrm{~d} t \\
& -\iint_{\Pi_{T}} H_{0}(u-M) A(u)_{x} \xi_{x} \mathrm{~d} x \mathrm{~d} t .
\end{aligned}
$$

Assume now that $\rho_{n}=\rho_{n}(x)$ is a standard sequence of mollifier functions in $\mathbb{R}$, and let us define $|u-m|_{n}^{+}:=|u-m|^{+} * \rho_{n}$ and $|u-M|_{n}^{+}:=|u-M|^{+} * \rho_{n}$ for $n \in \mathbb{N}$. Now we select the function $\zeta=\zeta(x, t)$ defined by

$$
\zeta=\zeta_{n, \varepsilon}:=H_{\varepsilon}\left(|u-m|_{n}^{+}+m-s-|u-M|_{n}^{+}\right), \quad s \in[m, M] .
$$


Let us denote the versions of $I_{p}$ obtained by replacing $|\cdot|^{+}$by $|\cdot|_{n}^{+}$and $\zeta=\zeta_{n, \varepsilon}$ by $I_{p}(n, \varepsilon), p=1, \ldots, 4$. Since $m$ and $s$ are constant and $\xi \zeta_{n, \varepsilon}$ has compact support, we get after an integration by parts

$$
\begin{aligned}
I_{1}(n, \varepsilon)=\iint_{\Pi_{T}}\left\{\left(|u-m|_{n}^{+}+m-s-|u-M|_{n}^{+}\right)\left(\xi \zeta_{n, \varepsilon}\right)_{t}+|u-M|_{n}^{+} \xi_{t}\right\} \mathrm{d} x \mathrm{~d} t \\
=\iint_{\Pi_{T}}\left\{-H_{\varepsilon}\left(|u-m|_{n}^{+}+m-s-|u-M|_{n}^{+}\right)\right. \\
\left.\quad \times\left(|u-m|_{n}^{+}+m-s-|u-M|_{n}^{+}\right)_{t} \xi+|u-M|_{n}^{+} \xi_{t}\right\} \mathrm{d} x \mathrm{~d} t .
\end{aligned}
$$

Taking $\varepsilon \downarrow 0$ and again integrating by parts yields

$$
\begin{aligned}
I_{1}(n, 0) & =\iint_{\Pi_{T}}\left\{-\left(|| u-\left.m\right|_{n} ^{+}+m-s-\left.|u-M|_{n}^{+}\right|^{+}\right)_{t} \xi+|u-M|_{n}^{+} \xi_{t}\right\} \mathrm{d} x \mathrm{~d} t \\
& =\iint_{\Pi_{T}}\left\{|| u-\left.m\right|_{n} ^{+}+m-s-\left.|u-M|_{n}^{+}\right|^{+} \xi_{t}+|u-M|_{n}^{+} \xi_{t}\right\} \mathrm{d} x \mathrm{~d} t,
\end{aligned}
$$

and letting $n \rightarrow \infty$ we find that $|u-m|_{n}^{+}+m-s-|u-M|_{n}^{+}$converges to $|u-m|^{+}+$ $m-s-|u-M|^{+}$in $L^{1}(\mathbb{R})$ and $\zeta_{n, 0}=H_{0}\left(|u-m|_{n}^{+}+m-s-|u-M|_{n}^{+}\right)$, or at least a subsequence, converges weak-* to some $\tilde{H}$ in $L^{\infty}\left(\Pi_{T}\right)$. Since $H$ is maximal monotone, it follows that $\tilde{H} \in H\left(|u-m|^{+}+m-s-|u-M|^{+}\right)$. Noting that $H(w) w=H_{0}(w) w$ for any function $w$, we arrive at

$$
I_{1}=\iint_{\Pi_{T}}\left\{|| u-\left.m\right|^{+}+m-s-\left.|u-M|^{+}\right|^{+}+|u-M|^{+}\right\} \xi_{t} \mathrm{~d} x \mathrm{~d} t=\iint_{\Pi_{T}}|u-s|^{+} \xi_{t} \mathrm{~d} x \mathrm{~d} t .
$$

Next, we deal with $I_{4}$. Since $A^{\prime}(u)=0$ for $u \in[m, M]$, we have

$$
H_{0}(u-M) A(u)_{x}=H_{0}(u-s) A(u)_{x}=H_{1}(u-m) A(u)_{x}
$$

for all $s \in[m, M]$, which gives

$$
I_{4}=\lim _{n \rightarrow \infty} \lim _{\varepsilon \downarrow 0} I_{4}(n, \varepsilon)=-\int_{\Pi_{T}} H_{0}(u-s) A(u)_{x} \xi_{x} \mathrm{~d} x \mathrm{~d} t .
$$

To deal with $I_{2}$, we proceed in a similar way as for $I_{1}$. We get

$$
\begin{aligned}
I_{2}(n, \varepsilon)= & \iint_{\Pi_{T}}\left\{\alpha\left(|u-m|_{n}^{+}+m-s-|u-M|_{n}^{+}\right)\left(\xi \zeta_{n, \varepsilon}\right)_{x}\right. \\
& \left.+(s-m) \alpha\left(\xi \zeta_{n, \varepsilon}\right)_{x}+\alpha|u-M|_{n}^{+} \xi_{x}\right\} \mathrm{d} x \mathrm{~d} t \\
= & -\iint_{\Pi_{T}} \alpha_{x} H_{\varepsilon}\left(|u-m|_{n}^{+}+m-s-|u-M|_{n}^{+}\right) \\
& \times\left(|u-m|_{n}^{+}+m-s-|u-M|_{n}^{+}\right) \xi \mathrm{d} x \mathrm{~d} t \\
& -\iint_{\Pi_{T}} \alpha\left(|u-m|_{n}^{+}+m-s-|u-M|_{n}^{+}\right)_{x} \\
& +\iint_{\Pi_{T}} \alpha|u-M|_{n}^{+} \xi_{x} \mathrm{~d} x \mathrm{~d} t+\iint_{\Pi_{T}} \alpha(s-m)\left(\xi \zeta_{n, \varepsilon}\right)_{x} \mathrm{~d} x \mathrm{~d} t .
\end{aligned}
$$


Taking $\varepsilon \downarrow 0$ we get, after integration by parts,

$$
\begin{aligned}
I_{2}(n, 0)= & -\iint_{\Pi_{T}} \alpha_{x}|| u-\left.m\right|_{n} ^{+}+m-s-\left.|u-M|_{n}^{+}\right|^{+} \xi \mathrm{d} x \mathrm{~d} t \\
& -\iint_{\Pi_{T}} \alpha\left(|| u-\left.m\right|_{n} ^{+}+m-s-\left.|u-M|_{n}^{+}\right|^{+}\right){ }_{x} \xi \mathrm{d} x \mathrm{~d} t \\
& +\iint_{\Pi_{T}} \alpha|u-M|_{n}^{+} \xi_{x} \mathrm{~d} x \mathrm{~d} t+\lim _{\varepsilon \downarrow 0} \iint_{\Pi_{T}} \alpha(s-m)\left(\xi \zeta_{n, \varepsilon}\right)_{x} \mathrm{~d} x \mathrm{~d} t \\
= & -\iint_{\Pi_{T}} \alpha_{x}|| u-\left.m\right|_{n} ^{+}+m-s-\left.|u-M|_{n}^{+}\right|^{+} \xi \mathrm{d} x \mathrm{~d} t \\
& +\iint_{\Pi_{T}} \alpha_{x}|| u-\left.m\right|_{n} ^{+}+m-s-\left.|u-M|_{n}^{+}\right|^{+} \xi \mathrm{d} x \mathrm{~d} t \\
& +\iint_{\Pi_{T}} \alpha|| u-\left.m\right|_{n} ^{+}+m-s-\left.|u-M|_{n}^{+}\right|^{+} \xi_{x} \mathrm{~d} x \mathrm{~d} t \\
& +\iint_{\Pi_{T}} \alpha|u-M|_{n}^{+} \xi_{x} \mathrm{~d} x \mathrm{~d} t+\lim _{\varepsilon \downarrow 0} \iint_{\Pi_{T}} \alpha(s-m)\left(\xi \zeta_{n, \varepsilon}\right)_{x} \mathrm{~d} x \mathrm{~d} t,
\end{aligned}
$$

where the two first terms obviously cancel. Sending $n \rightarrow \infty$ and proceeding like in the case $I_{1}$ we arrive at

$$
\begin{aligned}
I_{2}= & \iint_{\Pi_{T}} \alpha\left(|u-M|^{+}+|| u-\left.m\right|^{+}+m-s-\left.|u-M|^{+}\right|^{+}\right) \xi_{x} \mathrm{~d} x \mathrm{~d} t \\
& +\lim _{\substack{\varepsilon \downarrow 0 \\
n \rightarrow \infty}} \iint_{\Pi_{T}}(s-m) \alpha\left(\xi \zeta_{n, \varepsilon}\right)_{x} \mathrm{~d} x \mathrm{~d} t \\
= & \iint_{\Pi_{T}} \alpha|u-s|^{+} \xi_{x} \mathrm{~d} x \mathrm{~d} t+\lim _{\substack{\varepsilon \downarrow 0 \\
n \rightarrow \infty}} \iint_{\Pi_{T}}(s-m) \alpha\left(\xi \zeta_{n, \varepsilon}\right)_{x} \mathrm{~d} x \mathrm{~d} t .
\end{aligned}
$$

The last term of the last expression will be incorporated into the analysis of $I_{3}$. In fact, taking into account that

$$
\begin{aligned}
I_{3}(n, \varepsilon)=\iint_{\Pi_{T}} \alpha_{x}\left\{\left(H_{0}(u-M) M-H_{1}(u-m) m\right)\right. \\
\left.\quad \times H_{\varepsilon}\left(|u-m|_{n}^{+}+m-s-|u-M|_{n}^{+}\right)-H_{0}(u-M) M\right\} \xi \mathrm{d} x \mathrm{~d} t
\end{aligned}
$$

and that

$$
\lim _{\substack{\varepsilon \downarrow 0 \\ n \rightarrow \infty}} \iint_{\Pi_{T}}(s-m) \alpha\left(\xi \zeta_{n, \varepsilon}\right)_{x} \mathrm{~d} x \mathrm{~d} t=-\lim _{\substack{\varepsilon \downarrow 0 \\ n \rightarrow \infty}} \iint_{\Pi_{T}}(s-m) \alpha_{x} \xi \zeta_{n, \varepsilon} \mathrm{d} x \mathrm{~d} t,
$$

we obtain

$$
\begin{aligned}
& I_{3}+\lim _{\substack{\varepsilon \downarrow 0 \\
n \rightarrow \infty}} \iint_{\Pi_{T}}(s-m) \alpha\left(\xi \zeta_{n, \varepsilon}\right)_{x} \mathrm{~d} x \mathrm{~d} t \\
= & \lim _{\substack{\varepsilon \downarrow 0 \\
n \rightarrow \infty}}\left(I_{3}(n, \varepsilon)-\iint_{\Pi_{T}}(s-m) \alpha_{x} \xi \zeta_{n, \varepsilon} \mathrm{d} x \mathrm{~d} t\right) \\
= & \lim _{\substack{\varepsilon \downarrow 0 \\
n \rightarrow \infty}} \iint_{\Pi_{T}}\left\{\alpha_{x}\left(H_{0}(u-M) M-s+m-H_{1}(u-m) m\right) \xi \zeta_{n, \varepsilon}\right. \\
& \left.-\alpha_{x} H_{0}(u-M) M \xi\right\} \mathrm{d} x \mathrm{~d} t .
\end{aligned}
$$


Proceeding as in the cases $I_{1}$ and $I_{2}$ we find

$$
\begin{aligned}
I_{3}+\lim _{\substack{\varepsilon \downarrow 0 \\
n \rightarrow \infty}} \iint_{\Pi_{T}}(s-m) \alpha\left(\xi \zeta_{n, \varepsilon}\right)_{x} \mathrm{~d} x \mathrm{~d} t \\
=\iint_{\Pi_{T}} \alpha_{x}\left\{\left(H_{0}(u-M) M+m-s-H_{1}(u-m) m\right)\right. \\
\left.\quad \times \tilde{H}\left(|u-m|^{+}+m-s-|u-M|^{+}\right)-H_{0}(u-M) M\right\} \xi \mathrm{d} x \mathrm{~d} t .
\end{aligned}
$$

If $u>M$, then the expression in curled brackets in the last integrand equals

$$
\{\ldots\}=(M-s) \tilde{H}(M-s)-M=-s=-\tilde{H}(u-s) s .
$$

Likewise, for each of the cases $M \geq u>s>m, M>s \geq u>m$, and $M>s>u \geq m$ we verify that $\{\ldots\}=-\tilde{H}(u-s) s$, and also for $m>u$ we obtain

$$
\{\ldots\}=(m-s) \tilde{H}(m-s)=0=-\tilde{H}(u-s) s,
$$

and finally, the result is also valid if $s=m$ or $s=M$. We therefore conclude that

$$
I_{3}+\lim _{\substack{\varepsilon \downarrow 0 \\ n \rightarrow \infty}} \iint_{\Pi_{T}}(s-m) \alpha\left(\xi \zeta_{n, \varepsilon}\right)_{x} \mathrm{~d} x \mathrm{~d} t=-\int_{\Pi_{T}} \alpha_{x} \tilde{H}(u-s) s \xi \mathrm{d} x \mathrm{~d} t .
$$

Now, combining (2.10)-(2.13), we obtain the inequality

$$
\begin{aligned}
\iint_{\Pi_{T}}\left\{|u-s|^{+}\left(\xi_{t}+\alpha(x, t) \xi_{x}\right)-\alpha_{x}(x, t) \tilde{H}(u-s) s \xi\right. \\
\left.\quad-H_{0}(u-s) A(u)_{x} \xi_{x}\right\} \mathrm{d} x \mathrm{~d} t \geq 0 \quad \text { for all } s \in[m, M] .
\end{aligned}
$$

Now, for any $s \in[m, M)$ there exists a sequence $\left\{s_{n}\right\}_{n \in \mathbb{N}}$ such that $s_{n}<s<M$ and $s_{n} \rightarrow s$. Then $\tilde{H}\left(u-s_{n}\right) \rightarrow H_{0}(u-s)$ and $H_{0}\left(u-s_{n}\right) \rightarrow H_{0}(u-s)$ almost everywhere. Hence we get

$$
\begin{aligned}
\iint_{\Pi_{T}}\left\{|u-s|^{+}\left(\xi_{t}+\alpha(x, t) \xi_{x}\right)-\alpha_{x}(x, t) H_{0}(u-s) s \xi\right. \\
\left.\quad-H_{0}(u-s) A(u)_{x} \xi_{x}\right\} \mathrm{d} x \mathrm{~d} t \geq 0 \quad \text { for all } s \in[m, M] .
\end{aligned}
$$

This proof uses only that $\alpha_{x}$ is bounded, which is indeed the case for our choice $\alpha(x, t)=\Phi^{\prime}(v(x, t))$, since $\Phi$ is assumed to be smooth and $v_{x}(x, t)=u(x, t)$ is bounded. On the other hand, considering in the weak formulation a test function $\phi(x, t)=$ $H_{\varepsilon}(A(k)-A(u)) \varphi(x, t)$ with $k \notin[m, M]$ and following essentially the same steps as before we find

$$
\begin{aligned}
\iint_{\Pi_{T}}\left\{|s-u|^{+}\left(\xi_{t}+\alpha(x, t) \xi_{x}\right)+\alpha_{x}(x, t) H_{0}(s-u) s \xi\right. \\
\left.\quad+H_{0}(s-u) A(u)_{x} \xi_{x}\right\} \mathrm{d} x \mathrm{~d} t \geq 0 \quad \text { for all } s \in[m, M] .
\end{aligned}
$$

Adding (2.14) and (2.15) we get

$$
\begin{aligned}
\iint_{\Pi_{T}}\{ & |u-s|\left(\xi_{t}+\alpha(x, t) \xi_{x}\right)-\alpha_{x}(x, t) \operatorname{sgn}(u-s) s \xi \\
& \left.\quad-\operatorname{sgn}(u-s) A(u)_{x} \xi_{x}\right\} \mathrm{d} x \mathrm{~d} t \geq 0 \quad \text { for all } s \in[m, M] .
\end{aligned}
$$

Moreover (2.16) is valid for all $s \notin[m, M]$ (cf. [22]). This implies that any weak solution is an entropy solution. 


\section{Jump conditions and uniqueness}

3.1. Rankine-Hugoniot condition. Assume that $u$ is a weak solution having a discontinuity at a point $\left(x_{0}, t_{0}\right) \in \Pi_{T}$ between the approximate limits $u^{+}$and $u^{-}$of $u$ taken with respect to $x>x_{0}$ and $x<x_{0}$, respectively. Standard results from the theory of strongly degenerate parabolic equations imply that such a discontinuity is possible only if $A(u)$ is flat for $u \in \mathcal{I}\left(u^{-}, u^{+}\right):=\left[\min \left\{u^{-}, u^{+}\right\}, \max \left\{u^{-}, u^{+}\right\}\right]$. In that case, the propagation velocity of the jump is given by the Rankine-Hugoniot condition, which is derived by standard arguments from the weak formulation (2.2):

$$
s=\frac{1}{u^{+}-u^{-}}\left(\Phi^{\prime}\left(v^{+}\right) u^{+}-\Phi^{\prime}\left(v^{-}\right) u^{-}-\left(A(u)_{x}\right)^{+}+\left(A(u)_{x}\right)^{-}\right) .
$$

Here, $\left(A(u)_{x}\right)^{+}$and $\left(A(u)_{x}\right)^{-}$denote the approximate limits of $A(u)_{x}$ taken with respect to $x>x_{0}$ and $x<x_{0}$, respectively, and $v^{+}$and $v^{-}$denote the corresponding limits of $v$. Since $v$ is continuous, we actually have $v^{+}=v^{-}$, and (3.1) reduces to

$$
s=\Phi^{\prime}\left(v\left(x_{0}, t_{0}\right)\right)-\frac{\left(A(u)_{x}\right)^{+}-\left(A(u)_{x}\right)^{-}}{u^{+}-u_{-}} .
$$

3.2. Uniqueness of weak solutions. The uniqueness of weak solutions is an immediate consequence of Lemma 2.2 and a result proved in [22] (cf. also [15]) regarding continuous dependence of entropy solutions with respect to the flux function. More precisely, we have the following theorem.

THEOREM 3.1. Let $u$ and $\bar{u}$ be two weak solutions of (1.1), (1.2) (in the sense of Definition 2.1) with initial data $u_{0}$ and $\bar{u}_{0}$, respectively. Then there exists a constant $C=C\left(\max \left|\Phi^{\prime}\right|\right)$ such that

$$
\|u(\cdot, t)-\bar{u}(\cdot, t)\|_{L^{1}(\mathbb{R})} \leq C\left\|u_{0}-\bar{u}_{0}\right\|_{L^{1}(\mathbb{R})}, \quad \forall t \in(0, T] .
$$

In particular, weak solutions of (1.1), (1.2) are unique.

Proof. According to Lemma 2.2, $u$ and $\bar{u}$ are entropy solutions (in the sense of Definition 2.2) with initial data $u_{0}$ and $\bar{u}_{0}$, respectively. To be able to apply the $L^{1}$ stability and uniqueness results from [15, 22], we rewrite the equations satisfied by $u$ and $\bar{u}$ as

$$
u_{t}+(V(x, t) u)_{x}=A(u)_{x x}, \quad V(x, t):=\Phi^{\prime}\left(\int_{-\infty}^{x} u(y, t) \mathrm{d} y\right),
$$

with initial data $u(0, x)=u_{0}(x)$ and

$$
\bar{u}_{t}+(\bar{V}(x, t) \bar{u})_{x}=A(\bar{u})_{x x}, \quad \bar{V}(x, t):=\Phi^{\prime}\left(\int_{-\infty}^{x} \bar{u}(y, t) \mathrm{d} y\right) .
$$

with initial data $\bar{u}(0, x)=\bar{u}_{0}(x)$, respectively. Keeping in mind that $u$ and $\bar{u}$ are of bounded variation, i.e., $u, \bar{u} \in L^{\infty}(0, T ; B V(\mathbb{R}))$, we now may apply Theorem 1.3 in $[22]$ to conclude that there exists a constant $C$ such that

$$
\begin{aligned}
\|u(\cdot, t)-\bar{u}(\cdot, t)\|_{L^{1}(\mathbb{R})} & \leq\left\|u_{0}-\bar{u}_{0}\right\|_{L^{1}(\mathbb{R})}+\int_{0}^{t}\left|V_{x}(x, s)-\bar{V}_{x}(x, s)\right| \mathrm{d} s \\
& +\int_{0}^{t}|V(x, s)-\bar{V}(x, s)| \operatorname{TV}(u(\cdot, s)) \mathrm{d} s \\
& \leq\left\|u_{0}-\bar{u}_{0}\right\|_{L^{1}(\mathbb{R})}+C \int_{0}^{t}\left|V_{x}(x, s)-\bar{V}_{x}(x, s)\right| \mathrm{d} s .
\end{aligned}
$$


Observe that

$$
\int_{0}^{t}\left|V_{x}(x, s)-\bar{V}_{x}(x, s)\right| \mathrm{d} s \leq \max \left|\Phi^{\prime}\right| \int_{0}^{t}|u(x, s)-\bar{u}(x, s)| \mathrm{d} s,
$$

so that by the Gronwall inequality we arrive at

$$
\|u(\cdot, t)-\bar{u}(\cdot, t)\|_{L^{1}(\mathbb{R})} \leq \exp \left(\max \left|\Phi^{\prime}\right| t\right)\left\|u_{0}-\bar{u}_{0}\right\|_{L^{1}(\mathbb{R})} .
$$

\section{Convergence analysis of numerical schemes}

4.1. Preliminaries. We define the vectors $U^{n}:=\left\{u_{j+1 / 2}^{n}\right\}_{j \in \mathbb{Z}}$ and $V^{n}:=\left\{v_{j}^{n}\right\}_{j \in \mathbb{Z}}$, and discretize $\mathbb{R}$ by $x_{j}:=j \Delta x, j \in \mathbb{Z}$, and the time interval $[0, T]$ by $t_{n}=n \Delta t, n=0, \ldots, N, \Delta t:=T / N, N \in \mathbb{N}$. We denote by $u_{j+1 / 2}^{n}$ the cell average over $I_{j}:=\left[x_{j}, x_{j+1}\right]$ at time $t_{n}$ and $j \in \mathbb{Z}$. We also define $\lambda:=\Delta t / \Delta x$ and $\mu:=\Delta t / \Delta x^{2}=\lambda / \Delta x$ and wherever convenient use the spatial difference operators $\Delta_{+} \phi_{j}:=\phi_{j+1}-\phi_{j}, \Delta_{-} \phi_{j}:=\phi_{j}-\phi_{j-1}$, and

$$
\Delta^{2} \phi_{j}:=\Delta_{+} \Delta_{-} \phi_{j}=\phi_{j+1}-2 \phi_{j}+\phi_{j-1}
$$

We assume that the initial datum $u_{0}$ is discretized via

$$
u_{j+1 / 2}^{0}:=\frac{1}{\Delta x} \int_{I_{j}} u_{0}(\xi) d \xi, \quad j \in \mathbb{Z}
$$

Moreover, we define the operator $\mathcal{S}_{\Delta x}$ and its inverse $\mathcal{S}_{\Delta x}^{-1}$ via

$$
\mathcal{S}_{\Delta x}\left(U^{n} ; j\right):=\Delta x \sum_{l=-\infty}^{j-1} u_{l+1 / 2}^{n}, \quad \mathcal{S}_{\Delta x}^{-1}\left(V^{n} ; j\right):=\frac{v_{j+1}^{n}-v_{j}^{n}}{\Delta x} .
$$

Clearly, $\mathcal{S}_{\Delta x}$ and $\mathcal{S}_{\Delta x}^{-1}$ are the discrete analogues of the integral and differential operators that convert $u\left(\cdot, t_{n}\right)$ into $v\left(\cdot, t_{n}\right)$ and vice versa, respectively. Since we assume that $u_{0}$ is compactly supported, the sum in (4.1) is actually finite.

The numerical scheme for the initial value problem (1.1), (1.2) can be compactly written as follows:

$$
U^{n+1}=\left[\mathcal{S}_{\Delta x}^{-1} \circ \mathcal{H} \circ \mathcal{S}_{\Delta x}\right] U^{n}, \quad n=0, \ldots, N-1,
$$

where the basic idea is to utilize a standard scheme of the form

$$
V^{n+1}=\mathcal{H}\left(V^{n}\right), \quad n=0, \ldots, N-1
$$

for approximate solutions of the local PDE (1.4), starting from the initial data

$$
v_{j}^{0}:=\Delta x \sum_{l=-\infty}^{j-1} u_{l+1 / 2}^{0}=\int_{-\infty}^{x_{j}} u_{0}(\xi) \mathrm{d} \xi, \quad j \in \mathbb{Z} .
$$

Clearly, if $C_{0}$ is the total mass defined in (1.10), then we have that

$$
0 \leq v_{j}^{0} \leq C_{0}, \quad v_{j}^{0} \leq v_{j+1}^{0} \quad \text { for all } j \in \mathbb{Z} .
$$


Let us emphasize here that (4.2) implies that

$$
U^{n}=\left[\mathcal{S}_{\Delta x}^{-1} \circ \mathcal{H} \circ \mathcal{S}_{\Delta x}\right]^{n} U^{0}=\left[\mathcal{S}_{\Delta x}^{-1} \circ \mathcal{H}^{n} \circ \mathcal{S}_{\Delta x}\right] U^{0} .
$$

This means that for the actual computation of $U^{n}$ from $U^{0}$, the operators $\mathcal{S}_{\Delta x}$ and $\mathcal{S}_{\Delta x}^{-1}$ need to be applied only once, and not for every time step.

To derive properties of the scheme (4.2), we first analyze the scheme (4.3), which is here given by the marching formula

$$
v_{j}^{n+1}=v_{j}^{n}-\lambda \Delta_{+}\left[h\left(v_{j-1}^{n}, v_{j}^{n}\right)-A\left(\Delta_{-} v_{j}^{n} / \Delta x\right)\right], \quad j \in \mathbb{Z}, \quad n=0,1,2, \ldots,
$$

where $\lambda$ is subject to the CFL condition stated below, and

$$
h(w, z):=\Phi(0)+\Phi_{+}(w)+\Phi_{-}(z)
$$

is the Engquist-Osher flux [17], where we define the functions

$$
\Phi_{+}(v):=\int_{0}^{v} \max \left\{0, \Phi^{\prime}(s)\right\} \mathrm{d} s, \quad \Phi_{-}(v):=\int_{0}^{v} \min \left\{0, \Phi^{\prime}(s)\right\} \mathrm{d} s .
$$

We assume that $\Delta t$ and $\Delta x$ satisfy the CFL stability condition

$$
2 \lambda \max _{v \in\left[0, C_{0}\right]}\left|\Phi^{\prime}(v)\right|+2 \mu \max _{u \in \mathbb{R}}|a(u)| \leq 1
$$

The scheme for $u$ can be written as

$$
u_{j+1 / 2}^{n+1}=u_{j+1 / 2}^{n}-\lambda \Delta_{+} G_{j}^{n}+\mu \Delta^{2} A\left(u_{j+1 / 2}^{n}\right), \quad j \in \mathbb{Z}, \quad n=0,1,2, \ldots,
$$

where we define

$$
G_{j}^{n}:=\frac{1}{\Delta x} \Delta_{+} h\left(v_{j-1}^{n}, v_{j}^{n}\right)=\frac{1}{\Delta x}\left(\int_{v_{j-1}^{n}}^{v_{j}^{n}} \Phi_{+}^{\prime}(s) \mathrm{d} s+\int_{v_{j}^{n}}^{v_{j+1}^{n}} \Phi_{-}^{\prime}(s) \mathrm{d} s\right) .
$$

For the ease of reference, we will refer to (4.5)-(4.7) and (4.6), (4.7), (4.9), (4.10) as " $v$-scheme" and " $u$-scheme", respectively. Both schemes are, in particular, conservative, so the total mass $C_{0}$ is preserved.

The $v$-scheme (4.5)-(4.7) is a special case of the scheme studied by Evje and Karlsen [19] for the more general doubly degenerate parabolic equation $v_{t}+\Phi(v)_{x}=$ $B\left(A\left(v_{x}\right)\right)_{x}$. While Evje and Karlsen prove that their scheme converges to an entropy solution of that equation, we are here only interested in the property that the scheme is monotone, therefore TVD and monotonicity preserving, and produces solutions for which the discrete analogue of $v_{x}$ is uniformly bounded. This makes it possible here to take finite differences of that scheme to generate the $u$-scheme for the nonlocal Equation (1.1) satisfied by $u=v_{x}$. The convergence of the $u$-scheme will be analyzed separately.

4.2. Uniform estimates on $\left\{v_{j}^{n}\right\}$ and $\left\{u_{j+1 / 2}^{n}\right\}$. We establish the compactness and regularity estimates on the discrete soutions $\left\{v_{j}^{n}\right\}$ and $\left\{u_{j+1 / 2}^{n}\right\}$ in a series of lemmas. In Lemma 4.1 we prove that the $v$-scheme is monotone, and derive from this that the numerical solution $\left\{v_{j}^{n}\right\}$ satisfies an $L^{1}$ Lipschitz continuity in time property (Lemma 4.2). This result, in combination with the unboundedness of $A(u)$ for $u \rightarrow \infty$, allows us to prove (in Lemma 4.3) a uniform $L^{\infty}$ bound for $\left\{u_{j+1 / 2}^{n}\right\}$. 
Then, in Lemma 4.4, we prove that the spatial total variation of $A\left(U^{n}\right)$ is uniformly bounded. With the help of Lemma 4.5, which states that the cell that includes $v^{*}$ can move at most one position to the left or the right in one time step, we are then able to show (Lemma 4.6) that the spatial total variation of $U^{n}$ is bounded uniformly with respect to the discretization parameters; the bound depends, however, on the final time $T$. Then, in Lemma 4.7, we prove that the solution $\left\{u_{j+1 / 2}^{n}\right\}$ is $L^{1}$ Hölder continuous in time. Finally, we establish in Lemmas 4.8 and $4.9 L^{2}$ inequalities related to spatial and temporal translates of $\left\{A\left(u_{j+1 / 2}^{n}\right)\right\}$. The series of lemmas then permits us to prove the main convergence result, Theorem 4.1, which states that the numerical solutions $\left\{u_{j+1 / 2}^{n}\right\}$ produced by the $u$-scheme indeed converge to the unique weak solution (under conditions, and in a sense made precise in the theorem).

While Lemmas 4.1 to 4.7 are based on the original CFL condition (4.8), we need to employ a strengthened condition ((4.22), stated in Lemma 4.8) to prove Lemmas 4.8 and 4.9 , and eventually Theorem 4.1. Finally, we mention that the proofs of Lemmas 4.1 to 4.3 follow the treatment in [19].

LEMma 4.1. Under the CFL condition (4.8), the v-scheme defined by (4.5)-(4.7) is monotone.

Proof. We rewrite the scheme (4.5) as

$$
v_{j}^{n+1}=\mathcal{H}\left(v_{j-1}^{n}, v_{j}^{n}, v_{j+1}^{n}\right)=: \mathcal{H}_{j}^{n}, \quad j \in \mathbb{Z}, \quad n=0,1, \ldots, N-1 .
$$

Since $a \geq 0$, we then have

$$
\frac{\partial \mathcal{H}_{j}^{n}}{\partial v_{j \pm 1}^{n}}=\mp \lambda \min \left\{0, \Phi^{\prime}\left(v_{j \pm 1}^{n}\right)\right\}+\mu a\left(\Delta_{ \pm} v_{j}^{n} / \Delta x\right) \geq 0,
$$

while the CFL condition (4.8) implies that

$$
\begin{aligned}
\frac{\partial \mathcal{H}_{j}^{n}}{\partial v_{j}^{n}} & =1-\lambda\left(\max \left\{0, \Phi^{\prime}\left(v_{j}^{n}\right)\right\}-\min \left\{0, \Phi^{\prime}\left(v_{j}^{n}\right)\right\}\right)-\mu \Delta_{+} a\left(\Delta_{-} v_{j}^{n} / \Delta x\right) \\
& =1-\lambda\left|\Phi^{\prime}\left(v_{j}^{n}\right)\right|-\mu \Delta_{+} a\left(\Delta_{-} v_{j}^{n} / \Delta_{x}\right) \geq 0 .
\end{aligned}
$$

As a monotone scheme, the scheme (4.5) is total variation diminishing (TVD) and monotonicity preserving. Since (4.5) represents an explicit three-point scheme, for a fixed discretization $(\Delta x, \Delta t)$ we will always have

$$
v_{j}^{n}=0 \quad \text { for } j<-\mathcal{K}, \quad v_{j}^{n}=C_{0} \quad \text { for } j>\mathcal{K},
$$

for a sufficiently large constant $\mathcal{K}>0$. Thus, we can state the following corollary.

Corollary 4.1. If (4.4) and the CFL condition (4.8) hold, then the numerical solution $\left\{v_{j}^{n}\right\}$ produced by the v-scheme (4.5)-(4.7) satisfies

$$
0 \leq v_{j}^{n} \leq C_{0}, \quad v_{j}^{n} \leq v_{j+1}^{n} \quad \text { for all } j \in \mathbb{Z}, n=1, \ldots, N .
$$

As a direct consequence, the numerical solution values $V^{n}=\left\{v_{j}^{n}\right\}_{j \in \mathbb{Z}}$ satisfy the (trivial) uniform total variation bound

$$
\operatorname{TV}\left(V^{n}\right)=\sum_{j \in \mathbb{Z}}\left|v_{j+1}^{n}-v_{j}^{n}\right|=C_{0} .
$$


Lemma 4.2. The numerical solution $\left\{v_{j}^{n}\right\}$ produced by the v-scheme (4.5)-(4.7) satisfies the $L^{1}$ Lipschitz continuity in time property, i.e., there exists a constant $C_{1}$, which is independent of $\Delta:=(\Delta x, \Delta t)$, such that

$$
\sum_{j \in \mathbb{Z}}\left|v_{j}^{n+1}-v_{j}^{n}\right| \leq C_{1} \lambda
$$

Proof. For $j \in \mathbb{Z}$, the quantity $w_{j}^{n+1 / 2}:=v_{j}^{n+1}-v_{j}^{n}$ satisfies

$$
\begin{aligned}
w_{j}^{n+3 / 2}-w_{j}^{n+1 / 2}= & -\lambda \Delta_{+}\left[h\left(v_{j-1}^{n+1}, v_{j}^{n+1}\right)-h\left(v_{j-1}^{n}, v_{j}^{n}\right)\right] \\
& +\lambda \Delta_{+}\left[A\left(\Delta_{-} v_{j}^{n+1} / \Delta x\right)-A\left(\Delta_{-} v_{j}^{n} / \Delta x\right)\right] .
\end{aligned}
$$

We define

$$
\theta(s):= \begin{cases}1 / s & \text { if } s \neq 0 \\ 0 & \text { otherwise }\end{cases}
$$

and the quantities

$$
\begin{aligned}
B_{j}^{n+1 / 2} & :=\left[h\left(v_{j-1}^{n}, v_{j}^{n+1}\right)-h\left(v_{j-1}^{n}, v_{j}^{n}\right)\right] \theta\left(v_{j}^{n+1}-v_{j}^{n}\right), \\
C_{j}^{n+1 / 2}: & :=\left[h\left(v_{j}^{n+1}, v_{j+1}^{n+1}\right)-h\left(v_{j}^{n}, v_{j+1}^{n+1}\right)\right] \theta\left(v_{j}^{n+1}-v_{j}^{n}\right), \\
D_{j}^{n+1 / 2} & :=\left[A\left(\Delta_{+} v_{j}^{n+1} / \Delta x\right)-A\left(\Delta_{+} v_{j}^{n} / \Delta x\right)\right] \theta\left(\Delta_{+} v_{j}^{n+1}-\Delta_{+} v_{j}^{n}\right) .
\end{aligned}
$$

Since $h$ is a monotonically non-decreasing function of its first argument and a monotonically non-increasing function of its second argument, and $A$ is a monotonically non-decreasing function, we have

$$
C_{j}^{n+1 / 2} \geq 0, \quad D_{j}^{n+1 / 2} \geq 0, \quad B_{j}^{n+1 / 2} \leq 0 .
$$

After some manipulations and using (4.12) we obtain, from (4.14),

$$
\begin{aligned}
w_{j}^{n+3 / 2}= & w_{j}^{n+1 / 2}\left[1-\lambda C_{j}^{n+1 / 2}+\lambda B_{j}^{n+1 / 2}-\lambda\left(D_{j-1}^{n+1 / 2}+D_{j}^{n+1 / 2}\right)\right] \\
& +w_{j-1}^{n+1 / 2} \lambda\left(C_{j-1}^{n+1 / 2}+D_{j-1}^{n+1 / 2}\right)+w_{j+1}^{n+1 / 2} \lambda\left(-B_{j+1}^{n+1 / 2}+D_{j}^{n+1 / 2}\right) .
\end{aligned}
$$

Using the CFL condition we find

$$
\begin{aligned}
\left|w_{j}^{n+3 / 2}\right| \leq & \left|w_{j}^{n+1 / 2}\right|\left[1-\lambda\left(C_{j}^{n+1 / 2}-B_{j}^{n+1 / 2}+D_{j-1}^{n+1 / 2}+D_{j}^{n+1 / 2}\right)\right] \\
& +\left|w_{j-1}^{n+1 / 2}\right| \lambda\left(C_{j-1}^{n+1 / 2}+D_{j-1}^{n+1 / 2}\right)+\left|w_{j+1}^{n+1 / 2}\right| \lambda\left(-B_{j+1}^{n+1 / 2}+D_{j}^{n+1 / 2}\right) .
\end{aligned}
$$

Summing this over $j \in \mathbb{Z}$, and using (4.16) and (4.12), we obtain

$$
\sum_{j \in \mathbb{Z}}\left|w_{j}^{n+3 / 2}\right| \leq \sum_{j \in \mathbb{Z}}\left|w_{j}^{n+1 / 2}\right|
$$

which implies that

$$
\sum_{j \in \mathbb{Z}}\left|w_{j}^{n+3 / 2}\right| \leq \sum_{j \in \mathbb{Z}}\left|w_{j}^{1 / 2}\right|
$$


From (4.5) with $n=0$ we get

$$
\sum_{j \in \mathbb{Z}}\left|w_{j}^{1 / 2}\right|=\sum_{j \in \mathbb{Z}}\left|v_{j}^{1}-v_{j}^{0}\right|=\sum_{j \in \mathbb{Z}} \lambda\left|\Delta_{+}\left(h\left(v_{j-1}^{0}, v_{j}^{0}\right)-A\left(\Delta_{-} v_{j}^{0} / \Delta x\right)\right)\right| .
$$

Using (1.6) we arrive at (4.13).

Lemma 4.3. The numerical solution $\left\{v_{j}^{n}\right\}$ produced by the $v$-scheme (4.5)-(4.7) satisfies the inequality $\left|\Delta_{+} v_{j}^{n} / \Delta x\right| \leq C_{3}$ with a constant $C_{3}$, which is independent of $\Delta$. Equivalently, the solution $\left\{u_{j+1 / 2}^{n}\right\}$ generated by the u-scheme (4.6), (4.7), (4.9), (4.10) satisfies the uniform $L^{\infty}$ bound

$$
\left|u_{j+1 / 2}^{n}\right| \leq C_{3} \quad \text { for all } j \in \mathbb{Z}, n=0, \ldots, N .
$$

Proof. It is sufficient to show that $A\left(\Delta_{+} v_{j}^{n} / \Delta x\right) \leq C_{2}$ for a constant $C_{2}$ that is independent of $\Delta$. Taking into account (4.11) we get

$$
\begin{aligned}
& \left|A\left(\Delta_{+} v_{j}^{n} / \Delta x\right)\right|-\left|h\left(v_{j}^{n}, v_{j+1}^{n}\right)\right| \\
\leq & \left|A\left(\Delta_{+} v_{j}^{n} / \Delta x\right)-h\left(v_{j}^{n}, v_{j+1}^{n}\right)\right| \\
= & \left|\Phi(0)+\sum_{k=-\infty}^{j} \Delta_{-}\left(A\left(\Delta_{+} v_{k}^{n} / \Delta x\right)-h\left(v_{k}^{n}, v_{k+1}^{n}\right)\right)\right| \\
= & \left|\sum_{k=-\infty}^{j} \frac{v_{k}^{n+1}-v_{k}^{n}}{\lambda}+\Phi(0)\right| \leq \frac{1}{\lambda} \sum_{k \in \mathbb{Z}}\left|v_{k}^{n+1}-v_{k}^{n}\right|+|\Phi(0)| .
\end{aligned}
$$

Due to Lemma 4.2 , we see that $\left|A\left(\Delta_{+} v_{j}^{n} / \Delta x\right)\right| \leq C_{2}$ if we choose $C_{2}=C_{1}+|\Phi(0)|$. Taking into account (1.8) concludes the proof.

Lemma 4.4. The solution $\left\{u_{j+1 / 2}^{n}\right\}$ generated by the u-scheme (4.6), (4.7), (4.9), (4.10) satisfies the following inequality, where the constant $C_{4}$ is independent of $\Delta$ :

$$
\operatorname{TV}\left(A\left(U^{n}\right)\right)=\sum_{j \in \mathbb{Z}}\left|\Delta_{+} A\left(u_{j-1 / 2}^{n}\right)\right| \leq C_{4} .
$$

Proof. Using the marching formula (4.5) we can write

$$
\begin{aligned}
\left|\Delta_{+} A\left(u_{j-1 / 2}^{n}\right)\right| \leq & \frac{1}{\lambda}\left|v_{j}^{n+1}-v_{j}^{n}\right|+\left|\Delta_{+} h\left(v_{j-1}^{n}, v_{j}^{n}\right)\right| \\
\leq & \frac{1}{\lambda}\left|v_{j}^{n+1}-v_{j}^{n}\right|+\left|\left[h\left(v_{j}^{n}, v_{j+1}^{n}\right)-h\left(v_{j}^{n}, v_{j}^{n}\right)\right] \theta\left(v_{j+1}^{n}-v_{j}^{n}\right)\right|\left|\Delta_{+} v_{j}^{n}\right| \\
& +\left|\left[h\left(v_{j}^{n}, v_{j}^{n}\right)-h\left(v_{j-1}^{n}, v_{j}^{n}\right)\right] \theta\left(v_{j}^{n}-v_{j-1}^{n}\right)\right|\left|\Delta_{-} v_{j}^{n}\right| .
\end{aligned}
$$

Summing over $j \in \mathbb{Z}$ yields

$$
\sum_{j \in \mathbb{Z}}\left|\Delta_{+} A\left(u_{j-1 / 2}^{n}\right)\right| \leq \frac{1}{\lambda} \sum_{j \in \mathbb{Z}}\left|v_{j}^{n+1}-v_{j}^{n}\right|+2\left\|\Phi^{\prime}\right\|_{\infty} \sum_{j \in \mathbb{Z}}\left|\Delta_{+} v_{j}^{n}\right| .
$$

The right-hand side is uniformly bounded due to Lemma 4.2 and Corollary 4.1.

Lemma 4.4 does, in general, not permit to establish a uniform bound on the spatial total variation $\operatorname{TV}\left(U^{n}\right)$ of the solution values $\left\{u_{j+1 / 2}^{n}\right\}$ generated by the $u$-scheme. 
We now prove that $\operatorname{TV}\left(U^{n}\right)$ is nevertheless uniformly bounded, but by a bound that depends on the final time $T$. Our analysis will appeal to assumption (1.7). From (4.11) and (4.12) we deduce that if $v^{*}<C_{0}$, where we recall that $C_{0}$ is defined in (1.10), and $\left\{v_{j}^{n}\right\}$ is the numerical solution produced by the $v$-scheme (4.5)-(4.7), then at each time level there exists a unique index $k$ such that $v_{k}^{n}<v^{*} \leq v_{k+1}^{n}$. The following lemma informs about the behavior of this index with each time iteration. (In light of the discussion of Section 1.3, the case $v^{*}<C_{0}$ is the most relevant for the phenomenon of aggregation.)

Lemma 4.5. Assume that $v^{*}<C_{0}$, and that the data $\left\{v_{j}^{n}\right\}_{j \in \mathbb{Z}}$ and $\left\{v_{j}^{n+1}\right\}_{j \in \mathbb{Z}}$ have been produced by the $v$-scheme (4.5)-(4.7) starting from the monotone data $\left\{v_{j}^{0}\right\}_{j \in \mathbb{Z}}$ under the CFL condition (4.8). Let $k, \bar{k} \in \mathbb{Z}$ be the uniquely defined indices that satisfy $v_{k}^{n}<v^{*} \leq v_{k+1}^{n}$ and $v_{\bar{k}}^{n+1}<v^{*} \leq v_{\bar{k}+1}^{n+1}$, respectively. Then $\bar{k} \in\{k-1, k, k+1\}$.

Proof. Since $v_{k}^{n}<v^{*} \leq v_{k+1}^{n}$ we analyze two cases: $v_{k}^{n}<v^{*}<v_{k+1}^{n}$ and $v_{k}^{n}<v^{*}=$ $v_{k+1}^{n}$. In the first, the monotonicity of the $v$-scheme and (4.12) imply that

$$
v_{k-1}^{n+1} \leq v_{k}^{n}<v^{*}<v_{k+1}^{n} \leq v_{k+2}^{n+1},
$$

such that either $v_{k-1}^{n+1}<v^{*} \leq v_{k}^{n+1}$, or $v_{k}^{n+1}<v^{*} \leq v_{k+1}^{n+1}$, or $v_{k+1}^{n+1}<v^{*}<v_{k+2}^{n+1}$, which means that $\bar{k}=\{k-1, k, k+1\}$. In the second, we find that

$$
v_{k-1}^{n+1} \leq v_{k}^{n}<v^{*}=v_{k+1}^{n} \leq v_{k+2}^{n+1},
$$

so either $v_{k-1}^{n+1}<v^{*}=v_{k}^{n+1}$, or $v_{k}^{n+1}<v^{*} \leq v_{k+1}^{n+1}$, or $v_{k+1}^{n+1}<v^{*} \leq v_{k+2}^{n+1}$. We conclude the proof by noting that $v_{k+2}^{n+1}<v^{*}$ is impossible due to the monotonicity of the $v$-scheme and (4.12).

The next lemma states the announced bound on $\operatorname{TV}\left(U^{n}\right)$.

Lemma 4.6. Assume that the CFL condition (4.8) is satisfied. Then there exist constants $C_{5}$ and $C_{6}$, which are independent of $\Delta$, such that the solution values $U^{n}=\left\{u_{j+1 / 2}^{n}\right\}_{j \in \mathbb{Z}}$ satisfy the uniform total variation bound

$$
\operatorname{TV}\left(U^{n}\right)=\sum_{j \in \mathbb{Z}}\left|u_{j+1 / 2}^{n}-u_{j-1 / 2}^{n}\right| \leq\left(C_{5}+\mathrm{TV}\left(U^{0}\right)\right) \exp \left(C_{6} T\right), \quad n=1, \ldots, N
$$

Proof. From (4.9) we obtain

$$
\Delta_{+} u_{j-1 / 2}^{n+1}=\Delta_{+} u_{j-1 / 2}^{n}-\mu \Delta_{+} \Delta^{2} h\left(v_{j-1}^{n}, v_{j}^{n}\right)+\mu \Delta_{+} \Delta^{2} A\left(u_{j-1 / 2}^{n}\right) .
$$

Let us assume that $v^{*}<C_{0}$, so that there exists an index $k$ such that $v_{k}^{n}<v^{*} \leq v_{k+1}^{n}$ (cf. Lemma 4.5), and let us split $\mathbb{Z}$ into the subsets

$$
\begin{aligned}
& \mathcal{A}:=\mathcal{A}^{n}:=\{j \in \mathbb{Z} \mid j \leq k-2\}, \\
& \mathcal{B}:=\mathcal{B}^{n}:=\{j \in \mathbb{Z} \mid k-2<j \leq k+2\}, \\
& \mathcal{C}:=\mathcal{C}^{n}:=\{j \in \mathbb{Z} \mid k+2<j\} .
\end{aligned}
$$

(If $v^{*} \geq C_{0}$, the following arguments for $j \in \mathcal{A}$ apply to all $j \in \mathbb{Z}$, i.e. we may choose $\mathcal{A}=\mathbb{Z}$, and formally $\mathcal{B}=\mathcal{C}=\varnothing$.)

Let $w_{j}^{n}:=\Delta_{+} u_{j-1 / 2}^{n}$ and $a_{j}^{n}:=\Delta_{+} A\left(u_{j-1 / 2}^{n}\right) \theta\left(\Delta_{+} u_{j-1 / 2}^{n}\right)$. For $j \in \mathcal{A}$, we obtain

$$
w_{j}^{n+1}=w_{j}^{n}-\mu \Delta_{-} \Delta^{2} \Phi\left(v_{j}^{n}\right)+\mu \Delta^{2}\left(a_{j}^{n} w_{j}^{n}\right) .
$$


Using a Taylor expansion about $v_{j}^{n}$ we find that there exist numbers $\alpha_{j}^{n} \in\left[v_{j}^{n}, v_{j+1}^{n}\right]$ and $\beta_{j}^{n} \in\left[v_{j-1}^{n}, v_{j}^{n}\right]$ such that

$$
\Delta^{2} \Phi\left(v_{j}^{n}\right)=\Phi^{\prime}\left(v_{j}^{n}\right) w_{j}^{n} \Delta x+\frac{1}{2} \Phi^{\prime \prime}\left(\alpha_{j}^{n}\right)\left(\Delta_{+} v_{j}^{n}\right)^{2}+\frac{1}{2} \Phi^{\prime \prime}\left(\beta_{j}^{n}\right)\left(\Delta_{-} v_{j}^{n}\right)^{2} .
$$

Substituting this into (4.20) we obtain

$$
\begin{aligned}
w_{j}^{n+1}= & w_{j}^{n}-\lambda \Delta_{-}\left(\Phi^{\prime}\left(v_{j}^{n}\right) w_{j}^{n}\right)+\mu \Delta^{2}\left(a_{j}^{n} w_{j}^{n}\right)-\frac{\mu}{2} \Delta_{-}\left(\Phi^{\prime \prime}\left(\alpha_{j}^{n}\right)\left(\Delta_{+} v_{j}^{n}\right)^{2}\right) \\
& -\frac{\mu}{2} \Delta_{-}\left(\Phi^{\prime \prime}\left(\beta_{j}^{n}\right)\left(\Delta_{-} v_{j}^{n}\right)^{2}\right) \\
= & w_{j}^{n}-\lambda \Delta_{-}\left(\Phi^{\prime}\left(v_{j}^{n}\right) w_{j}^{n}\right)+\mu \Delta^{2}\left(a_{j}^{n} w_{j}^{n}\right) \\
& -\frac{\mu}{2}\left(\Delta_{-} \Phi^{\prime \prime}\left(\alpha_{j}^{n}\right)\left(\Delta_{+} v_{j}^{n}\right)^{2}+\Phi^{\prime \prime}\left(\alpha_{j-1}^{n}\right)\left(v_{j+1}^{n}-v_{j-1}^{n}\right) w_{j}^{n} \Delta x\right. \\
& \left.\quad+\Delta_{-} \Phi^{\prime \prime}\left(\beta_{j}^{n}\right)\left(\Delta_{-} v_{j}^{n}\right)^{2}+\Phi^{\prime \prime}\left(\beta_{j-1}^{n}\right)\left(v_{j}^{n}-v_{j-2}^{n}\right) w_{j-1}^{n} \Delta x\right) \\
= & w_{j}^{n}\left[1-\lambda \Phi^{\prime}\left(v_{j}^{n}\right)-2 \mu a_{j}^{n}\right]+w_{j-1}^{n}\left[\mu a_{j-1}^{n}+\lambda \Phi^{\prime}\left(v_{j-1}^{n}\right)\right]+\mu w_{j+1}^{n} a_{j+1}^{n} \\
& +\mathcal{O}(\Delta t)\left(w_{j-1}^{n}+w_{j}^{n}+\Delta_{+} v_{j}^{n}+\Delta_{-} v_{j}^{n}\right) .
\end{aligned}
$$

In an analogous way we find, for $j \in \mathcal{C}$,

$$
\begin{aligned}
w_{j}^{n+1}= & w_{j}^{n}\left[1+\lambda \Phi^{\prime}\left(v_{j}^{n}\right)-2 \mu a_{j}^{n}\right]+w_{j+1}^{n}\left[\mu a_{j+1}^{n}-\lambda \Phi^{\prime}\left(v_{j+1}^{n}\right)\right]+\mu w_{j-1}^{n} a_{j-1}^{n} \\
& +\mathcal{O}(\Delta t)\left(w_{j}^{n}+w_{j+1}^{n}+\Delta_{+} v_{j}^{n}+\Delta_{-} v_{j}^{n}\right) .
\end{aligned}
$$

Now we deal with $j \in \mathcal{B}$. For $j=k-1$, using that $v^{*}$ is a maximum of $\Phi$ and following analogous steps as before, we get

$$
\begin{aligned}
w_{k-1}^{n+1}= & w_{k-1}^{n}-\mu\left(\Phi\left(v_{k+1}^{n}\right)-\Phi\left(v^{*}\right)+\Delta_{-} \Delta^{2} \Phi\left(v_{k-1}^{n}\right)\right)+\mu \Delta^{2}\left(a_{k-1}^{n} w_{k-1}^{n}\right) \\
= & w_{k-1}^{n}-\mu\left(\Phi^{\prime}(\xi)\left(v_{k+1}^{n}-v^{*}\right)+\Delta_{-} \Delta^{2} \Phi\left(v_{k-1}^{n}\right)\right)+\mu \Delta^{2}\left(a_{k-1}^{n} w_{k-1}^{n}\right) \\
= & w_{k-1}^{n}-\mu\left(\left(\Phi^{\prime}(\xi)-\Phi^{\prime}\left(v^{*}\right)\right)\left(v_{k+1}^{n}-v^{*}\right)+\Delta_{-} \Delta^{2} \Phi\left(v_{k-1}^{n}\right)\right)+\mu \Delta^{2}\left(a_{k-1}^{n} w_{k-1}^{n}\right) \\
= & w_{k-1}^{n}\left[1-\lambda \Phi^{\prime}\left(v_{k-1}^{n}\right)-2 \mu a_{k-1}^{n}\right]+w_{k-2}^{n}\left[\mu a_{k-2}^{n}+\lambda \Phi^{\prime}\left(v_{k-2}^{n}\right)\right]+\mu w_{k}^{n} a_{k}^{n} \\
& +\mathcal{O}(\Delta t)\left(1+w_{k-2}^{n}+w_{k-1}^{n}+\Delta_{+} v_{k-1}^{n}+\Delta_{-} v_{k-1}^{n}\right) .
\end{aligned}
$$

For $j=k$, using that $\Phi^{\prime}\left(v^{*}\right)=0$ we compute

$$
\begin{aligned}
w_{k}^{n+1}= & w_{k}^{n}-\mu\left[\Phi\left(v_{k+2}^{n}\right)-2 \Phi\left(v_{k+1}^{n}\right)+\Phi\left(v_{k}^{n}\right)-\left\{\Phi\left(v_{k}^{n}\right)-2 \Phi\left(v_{k-1}^{n}\right)+\Phi\left(v_{k-2}^{n}\right)\right\}\right] \\
& -\mu\left[\Phi\left(v_{k-1}^{n}\right)-\Phi\left(v_{k}^{n}\right)+2\left(\Phi\left(v^{*}\right)-\Phi\left(v_{k}^{n}\right)\right)+\Phi\left(v^{*}\right)-\Phi\left(v_{k+1}^{n}\right)\right]+\mu \Delta^{2}\left(a_{k}^{n} w_{k}^{n}\right) \\
= & w_{k}^{n}-\mu\left[\Delta_{+} \Delta^{2} \Phi\left(v_{k}^{n}\right)+\Delta_{-} \Delta^{2} \Phi\left(v_{k}^{n}\right)\right]+\mu \Delta^{2}\left(a_{k}^{n} w_{k}^{n}\right) \\
& -\mu\left[\Phi\left(v_{k-1}^{n}\right)-\Phi\left(v_{k}^{n}\right)+2\left(\Phi\left(v^{*}\right)-\Phi\left(v_{k}^{n}\right)\right)+\Phi\left(v^{*}\right)-\Phi\left(v_{k+1}^{n}\right)\right] \\
= & w_{k}^{n}\left(1-2 \mu a_{k}^{n}\right)+w_{k-1}^{n}\left[\mu a_{k-1}^{n}+\lambda \Phi^{\prime}\left(v_{k-1}^{n}\right)\right]+w_{k+1}^{n}\left[\mu a_{k+1}^{n}-\lambda \Phi^{\prime}\left(v_{k+1}^{n}\right)\right] \\
& +\mathcal{O}(\Delta t)\left(1+w_{k-1}^{n}+w_{k}^{n}+w_{k+1}^{n}+\Delta_{+} v_{k}^{n}+\Delta_{-} v_{k}^{n}\right) .
\end{aligned}
$$

For $j=k+1$ and $j=k+2$, the following steps are analogous to the previous cases. 
Using that $\Phi^{\prime}\left(v^{*}\right)=0$ we obtain

$$
\begin{aligned}
w_{k+1}^{n+1}= & w_{k+1}^{n}-\mu\left[\Delta_{+} \Delta^{2} \Phi\left(v_{k+1}^{n}\right)+3\left(\Phi\left(v_{k}^{n}\right)-\Phi\left(v^{*}\right)\right)+\Phi\left(v_{k}^{n}\right)-\Phi\left(v_{k-1}^{n}\right)\right] \\
& +\mu \Delta^{2}\left(a_{k+1}^{n} w_{k+1}^{n}\right) \\
= & w_{k+1}^{n}\left[1+\lambda \Phi^{\prime}\left(v_{k+1}^{n}\right)-2 \mu a_{k+1}^{n}\right]+w_{k}^{n} \mu a_{k}^{n}+w_{k+2}^{n}\left[\mu a_{k+2}^{n}-\lambda \Phi^{\prime}\left(v_{k+2}^{n}\right)\right] \\
& +\mathcal{O}(\Delta t)\left(1+w_{k+1}^{n}+w_{k+2}^{n}+\Delta_{+} v_{k+1}^{n}+\Delta_{-} v_{k+1}^{n}\right), \\
w_{k+2}^{n+1}= & w_{k+2}^{n}-\mu\left[\Delta_{+} \Delta^{2} \Phi\left(v_{k+2}^{n}\right)+\Phi\left(v_{k}^{n}\right)-\Phi\left(v^{*}\right)\right]+\mu \Delta^{2}\left(a_{k+2}^{n} w_{k+2}^{n}\right) \\
= & w_{k+2}^{n}\left[1+\lambda \Phi^{\prime}\left(v_{k+2}^{n}\right)-2 \mu a_{k+2}^{n}\right]+w_{k+3}^{n}\left[\mu a_{k+3}^{n}-\lambda \Phi^{\prime}\left(v_{k+3}^{n}\right)\right]+\mu w_{k+1}^{n} a_{k+1}^{n} \\
& +\mathcal{O}(\Delta t)\left(1+w_{k+2}^{n}+w_{k+3}^{n}+\Delta_{+} v_{k+2}^{n}+\Delta_{-} v_{k+2}^{n}\right) .
\end{aligned}
$$

Finally, summing over $j$ we find that there exist constants $C_{6}$ and $C_{7}$ such that

$$
\sum_{j \in \mathbb{Z}}\left|w_{j}^{n+1}\right| \leq \sum_{j \in \mathbb{Z}}\left|w_{j}^{n}\right|\left(1+C_{6} \Delta t\right)+C_{7} \Delta t,
$$

which implies that

$$
\sum_{j \in \mathbb{Z}}\left|w_{j}^{n+1}\right| \leq \sum_{j \in \mathbb{Z}}\left|w_{j}^{0}\right| \exp \left(C_{6} T\right)+\frac{C_{7}}{C_{6}} \exp \left(C_{6} T\right),
$$

which proves (4.18).

The next lemma states $L^{1}$ Hölder continuity with respect to the variable $t$ of the solution generated by (4.9).

Lemma 4.7. The solution $\left\{u_{j+1 / 2}^{n}\right\}$ generated by the u-scheme (4.6), (4.7), (4.9), (4.10) satisfies the following inequality, where the constant $C_{8}$ is independent of $\Delta$ :

$$
\sum_{j \in \mathbb{Z}}\left|u_{j+1 / 2}^{m}-u_{j+1 / 2}^{n}\right| \Delta x \leq C_{8} \sqrt{\Delta t(m-n)} \text { for } m>n \text {, and } m, n \in \mathbb{N}_{0} .
$$

Proof. We first establish weak Lipschitz continuity in the time variable. To this end, let $\phi(x)$ be a test function and $\phi_{j}:=\phi(j \Delta x)$. Multiplying Equation (4.9) by $\phi_{j} \Delta x$, summing over $n$ and $j$ and applying a summation by parts, we get

$$
\begin{aligned}
\left|\Delta x \sum_{j \in \mathbb{Z}} \phi_{j}\left(u_{j+1 / 2}^{n+1}-u_{j+1 / 2}^{n}\right)\right| \leq & \left|\Delta t \sum_{j \in \mathbb{Z}} G_{j}^{n}\left(\phi_{j}-\phi_{j-1}\right)\right| \\
& +\left|\lambda \sum_{j \in \mathbb{Z}}\left(\phi_{j}-\phi_{j-1}\right)\left(A\left(u_{j+1 / 2}^{n}\right)-A\left(u_{j-1 / 2}^{n}\right)\right)\right| .
\end{aligned}
$$

Using Lemma 4.4 and the fact that $\phi$ is smooth we obtain

$$
\left|\Delta x \sum_{j \in \mathbb{Z}} \phi_{j}\left(u_{j+1 / 2}^{n+1}-u_{j+1 / 2}^{n}\right)\right| \leq C\left\|\phi^{\prime}\right\| \Delta t,
$$

where $C$ is independent of $\Delta$ and $\phi$. Consequently, for $m>n$ the following weak continuity result holds:

$$
\left|\Delta x \sum_{j \in \mathbb{Z}} \phi_{j}\left(u_{j+1 / 2}^{m}-u_{j+1 / 2}^{n}\right)\right| \leq C\left\|\phi^{\prime}\right\| \Delta t(m-n) .
$$


Since $E_{j}:=u_{j+1 / 2}^{m}-u_{j+1 / 2}^{n}$ has bounded variation on $\mathbb{R}$, we arrive at the inequality (4.21) by proceeding as in [18, Lemma 3.6].

Now, following the treatment in [21] we prove an $L^{2}$ estimate for the discrete version of $A(u)_{x}$.

LEMMA 4.8. Assume that the following strengthened CFL condition is satisfied for a constant $\varepsilon>0$ :

$$
\mathrm{CFL}_{\varepsilon}:=2 \lambda \max _{u \in \mathbb{R}}\left|\Phi^{\prime}(u)\right|+4 \mu \max _{u \in \mathbb{R}} a(u) \leq 1-\varepsilon .
$$

Then the solution $\left\{u_{j+1 / 2}^{n}\right\}$ generated by the u-scheme (4.9), (4.10) satisfies the following inequality, where the constant $C_{9}$ depends on $\varepsilon$, but is independent of $\Delta$ :

$$
\sum_{n=1}^{N} \sum_{j \in \mathbb{Z}}\left(\frac{\Delta_{-} A\left(u_{j+1 / 2}^{n}\right)}{\Delta x}\right)^{2} \Delta t \Delta x \leq C_{9} .
$$

Proof. Multiplying (4.9), by $u_{j+1 / 2}^{n} \Delta x$, summing the result over $n=0, \ldots, N-1$ and $j \in \mathbb{Z}$, and using summations by parts we get

$$
\begin{aligned}
& \lambda \sum_{n=0}^{N-1} \sum_{j \in \mathbb{Z}}\left(\Delta_{-} A\left(u_{j+1 / 2}^{n}\right)\right)\left(\Delta_{-} u_{j+1 / 2}^{n}\right) \\
= & \Delta t \sum_{n=0}^{N-1} \sum_{j \in \mathbb{Z}} G_{j}^{n}\left(\Delta_{-} u_{j+1 / 2}^{n}\right)-\frac{\Delta x}{2} \sum_{n=0}^{N-1} \sum_{j \in \mathbb{Z}}\left(\left(u_{j+1 / 2}^{n+1}\right)^{2}-\left(u_{j+1 / 2}^{n}\right)^{2}\right) \\
& +\frac{\Delta x}{2} \sum_{n=0}^{N-1} \sum_{j \in \mathbb{Z}}\left(u_{j+1 / 2}^{n+1}-u_{j+1 / 2}^{n}\right)^{2},
\end{aligned}
$$

where we used that

$$
\left(u_{j+1 / 2}^{n+1}-u_{j+1 / 2}^{n}\right) u_{j+1 / 2}^{n}=\frac{1}{2}\left[\left(u_{j+1 / 2}^{n+1}\right)^{2}-\left(u_{j+1 / 2}^{n}\right)^{2}-\left(u_{j+1 / 2}^{n+1}-u_{j+1 / 2}^{n}\right)^{2}\right] .
$$

In light of Lemma 4.3, we can also write

$$
\left(\Delta_{-} A\left(u_{j+1 / 2}^{n}\right)\right)\left(\Delta_{-} u_{j+1 / 2}^{n}\right) \geq \frac{1}{a^{*}}\left(\Delta_{-} A\left(u_{j+1 / 2}^{n}\right)\right)^{2}, \quad a^{*}:=\max _{u} a(u),
$$

since $a(u) \geq 0$. Using this observation, we find that

$$
\begin{aligned}
\frac{\lambda}{a^{*}} \sum_{n=0}^{N-1} \sum_{j \in \mathbb{Z}}\left(\Delta_{-} A\left(u_{j+1 / 2}^{n}\right)\right)^{2} \leq & \Delta t \sum_{n=0}^{N-1} \sum_{j \in \mathbb{Z}} G_{j}^{n}\left(\Delta_{-} u_{j+1 / 2}^{n}\right)+\frac{\Delta x}{2} \sum_{j \in \mathbb{Z}}\left(u_{j+1 / 2}^{0}\right)^{2} \\
& +\frac{\Delta x}{2} \sum_{n=0}^{N-1} \sum_{j \in \mathbb{Z}}\left(u_{j+1 / 2}^{n+1}-u_{j+1 / 2}^{n}\right)^{2} .
\end{aligned}
$$

On the other hand, from (4.9) and the inequality $(a+b)^{2} \leq 2 a^{2}+2 b^{2}$ we obtain

$$
\frac{1}{2}\left(u_{j+1 / 2}^{n+1}-u_{j+1 / 2}^{n}\right)^{2} \leq \lambda^{2}\left(\Delta_{+} G_{j}^{n}\right)^{2}+2 \mu^{2}\left(\left(\Delta_{+} A\left(u_{j+1 / 2}^{n}\right)\right)^{2}+\left(\Delta_{-} A\left(u_{j+1 / 2}^{n}\right)\right)^{2}\right) .
$$


Multiplying the last inequality by $\Delta x$ and summing the result over $n$ and $j$ yields

$$
\begin{aligned}
\frac{\Delta x}{2} \sum_{n=0}^{N-1} \sum_{j \in \mathbb{Z}}\left(u_{j+1 / 2}^{n+1}-u_{j+1 / 2}^{n}\right)^{2} \leq & \frac{\Delta t^{2}}{\Delta x} \sum_{n=0}^{N-1} \sum_{j \in \mathbb{Z}}\left(\Delta_{+} G_{j}^{n}\right)^{2} \\
& +4 \mu^{2} \Delta x \sum_{n=0}^{N-1} \sum_{j \in \mathbb{Z}}\left(\Delta_{-} A\left(u_{j+1 / 2}^{n}\right)\right)^{2} .
\end{aligned}
$$

The new CFL condition (4.22) now implies that

$$
4 \mu^{2} \Delta x=4 \mu \frac{\Delta t}{\Delta x} \leq \frac{\Delta t(1-\varepsilon)}{\Delta x a^{*}},
$$

and therefore

$$
\begin{aligned}
& \frac{\Delta x}{2} \sum_{n=0}^{N-1} \sum_{j \in \mathbb{Z}}\left(u_{j+1 / 2}^{n+1}-u_{j+1 / 2}^{n}\right)^{2} \\
\leq & \frac{\Delta t^{2}}{\Delta x} \sum_{n=0}^{N-1} \sum_{j \in \mathbb{Z}}\left(\Delta_{+} G_{j}^{n}\right)^{2}+\frac{\Delta t(1-\varepsilon)}{\Delta x a^{*}} \sum_{n=0}^{N-1} \sum_{j \in \mathbb{Z}}\left(\Delta_{-} A\left(u_{j+1 / 2}^{n}\right)\right)^{2} .
\end{aligned}
$$

Summing (4.24) and (4.25) yields

$$
\begin{aligned}
& \frac{\varepsilon \lambda}{a^{*}} \sum_{n=0}^{N-1} \sum_{j \in \mathbb{Z}}\left(\Delta_{-} A\left(u_{j+1 / 2}^{n}\right)\right)^{2} \\
\leq & \Delta t \sum_{n=0}^{N-1} \sum_{j \in \mathbb{Z}} G_{j}^{n}\left(\Delta_{-} u_{j+1 / 2}^{n}\right)+\frac{\Delta x}{2} \sum_{j \in \mathbb{Z}}\left(u_{j+1 / 2}^{0}\right)^{2}+\frac{\Delta t^{2}}{\Delta x} \sum_{n=0}^{N-1} \sum_{j \in \mathbb{Z}}\left(\Delta_{-} G_{j+1}^{n}\right)^{2} \leq C,
\end{aligned}
$$

where we used Lemma 4.6, the bound on $G_{j}^{n}$ and the fact that $\Delta t=\mathcal{O}\left(\Delta x^{2}\right)$.

With the help of Lemma 4.8 we can prove

LEMma 4.9. Under the assumptions of Lemma 4.8 there exists a constant $C_{10}$ which is independent of $\Delta$ such that

$$
\sum_{j \in \mathbb{Z}}\left|A\left(u_{j+1 / 2}^{m}\right)-A\left(u_{j+1 / 2}^{n}\right)\right|^{2} \Delta x \leq C_{10}(m-n) \Delta t \quad \text { for } m>n .
$$

Proof. Using Lemma 4.3, the fact that $A^{\prime}(u) \geq 0$, and (4.9), we get

$$
\begin{aligned}
& \sum_{j \in \mathbb{Z}}\left(A\left(u_{j+1 / 2}^{m}\right)-A\left(u_{j+1 / 2}^{n}\right)\right)^{2} \Delta x \\
\leq & a^{*} \sum_{j \in \mathbb{Z}}\left(A\left(u_{j+1 / 2}^{m}\right)-A\left(u_{j+1 / 2}^{n}\right)\right)\left(u_{j+1 / 2}^{m}-u_{j+1 / 2}^{n}\right) \Delta x=: \mathcal{A}+\mathcal{B},
\end{aligned}
$$

where we define

$$
\begin{aligned}
& \mathcal{A}:=-\Delta t a^{*} \sum_{j \in \mathbb{Z}}\left(A\left(u_{j+1 / 2}^{m}\right)-A\left(u_{j+1 / 2}^{n}\right)\right) \sum_{l=n}^{m-1} \Delta_{+} G_{j}^{l}, \\
& \mathcal{B}:=\lambda a^{*} \sum_{j \in \mathbb{Z}}\left(A\left(u_{j+1 / 2}^{m}\right)-A\left(u_{j+1 / 2}^{n}\right)\right) \sum_{l=n}^{m-1} \Delta^{2} A\left(u_{j+1 / 2}^{l}\right) .
\end{aligned}
$$


Summing by parts we get

$$
\mathcal{A}=\Delta t a^{*} \sum_{j \in \mathbb{Z}} \sum_{l=n}^{m-1} G_{j}^{l}\left(\Delta_{-} A\left(u_{j+1 / 2}^{m}\right)-\Delta_{-} A\left(u_{j+1 / 2}^{n}\right)\right) .
$$

We can write

$$
\mathcal{A}=\Delta t \Delta x a^{*} \sum_{j \in \mathbb{Z}} \sum_{l=n}^{m-1} G_{j}^{l}\left(\frac{\Delta_{-} A\left(u_{j+1 / 2}^{m}\right)}{\Delta x}-\frac{\Delta_{-} A\left(u_{j+1 / 2}^{n}\right)}{\Delta x}\right) .
$$

Using that $a b \leq a^{2}+b^{2}$, we find

$$
\begin{aligned}
\mathcal{A} \leq & \frac{\Delta t}{2} a^{*} \sum_{j \in \mathbb{Z}} \sum_{l=n}^{m-1}\left|G_{j}^{l}\right|\left[\left(\frac{\Delta_{-} A\left(u_{j+1 / 2}^{m}\right)}{\Delta x}\right)^{2}+\left(\frac{\Delta_{-} A\left(u_{j+1 / 2}^{n}\right)}{\Delta x}\right)^{2}\right] \Delta x \\
& +\Delta t a^{*} \sum_{j \in \mathbb{Z}} \sum_{l=n}^{m-1}\left|G_{j}^{l}\right| \Delta x=\mathcal{O}((m-n) \Delta t),
\end{aligned}
$$

where we have used Lemma 4.8 and the bound on $G_{j}^{n}$.

Proceeding in the same way for $\mathcal{B}$ yields

$$
\begin{aligned}
\mathcal{B}= & -\lambda a^{*} \sum_{j \in \mathbb{Z}}\left\{\left[A\left(u_{j+1 / 2}^{m}\right)-A\left(u_{j+1 / 2}^{n}\right)-\left(A\left(u_{j-1 / 2}^{m}\right)-A\left(u_{j-1 / 2}^{n}\right)\right)\right]\right. \\
& \left.\times \sum_{l=n}^{m-1} \Delta_{-} A\left(u_{j+1 / 2}^{l}\right)\right\} \\
= & -\lambda a^{*} \sum_{j \in \mathbb{Z}}\left\{\left(\Delta_{-} A\left(u_{j+1 / 2}^{m}\right)-\Delta_{-} A\left(u_{j+1 / 2}^{n}\right)\right) \sum_{l=n}^{m-1} \Delta_{-} A\left(u_{j+1 / 2}^{l}\right)\right\} \\
= & -\lambda a^{*} \sum_{j \in \mathbb{Z}} \sum_{l=n}^{m-1}\left(\Delta_{-} A\left(u_{j+1 / 2}^{m}\right) \cdot \Delta_{-} A\left(u_{j+1 / 2}^{l}\right)-\Delta_{-} A\left(u_{j+1 / 2}^{n}\right) \cdot \Delta_{-} A\left(u_{j+1 / 2}^{l}\right)\right) \\
\leq & 2(m-n) \Delta t a^{*} \sum_{j \in \mathbb{Z}}\left(\frac{\Delta_{-} A\left(u_{j+1 / 2}^{n}\right)}{\Delta x}\right)^{2} \Delta x=\mathcal{O}((m-n) \Delta t) .
\end{aligned}
$$

Inserting into (4.27) that $\mathcal{A}, \mathcal{B}=\mathcal{O}((m-n) \Delta t)$ concludes the proof.

Let us now denote by $u^{\Delta}$ the piecewise constant function

$$
u^{\Delta}(x, t):=\sum_{n=0}^{N-1} \sum_{j \in \mathbb{Z}} \chi_{j n}(x, t) u_{j+1 / 2}^{n},
$$

where $\chi_{j n}$ denotes the characteristic function of $I_{j} \times\left[t_{n}, t_{n+1}\right)$, and let us denote by $v^{\Delta}$ its primitive. From the $L^{\infty}$ bound (Lemma 4.3), the uniform bound on the total variation in space (Lemma 4.6), and the $L^{1}$ Hölder continuity in time result (Lemma 4.7) we infer that there is a constant $C$ such that

$$
\left\|u^{\Delta}\right\|_{L^{\infty}\left(\Pi_{T}\right)}+\left\|u^{\Delta}\right\|_{L^{1}\left(\Pi_{T}\right)} \leq C ; \quad\left|u^{\Delta}(\cdot, t)\right|_{B V(\mathbb{R})} \leq C \quad \text { for all } t \in(0, T],
$$


uniformly as $\Delta x, \Delta t \downarrow 0$, while Lemmas 4.8 and 4.9 imply (cf. [20]) that there are constants $C_{11}$ and $C_{12}$ independent of $\Delta$ such that

$$
\begin{aligned}
& \left\|A\left(u^{\Delta}(\cdot+y, \cdot)\right)-A\left(u^{\Delta}(\cdot, \cdot)\right)\right\|_{L^{2}\left(\Pi_{T}\right)} \leq C_{11} \sqrt{|y|(|y|+\Delta x)}, \\
& \left\|A\left(u^{\Delta}(\cdot, \cdot+\tau)\right)-A\left(u^{\Delta}(\cdot, \cdot)\right)\right\|_{L^{2}\left(\Pi_{T-\tau}\right)} \leq C_{12} \sqrt{\tau} .
\end{aligned}
$$

\subsection{Convergence to the weak solution.}

TheOrem 4.1. Assume that $\Delta x$ and $\Delta t$ satisfy the $C F L_{\varepsilon}$ condition (4.22), and that $u_{0}$ is compactly supported and satisfies (1.6). Then the piecewise constant solutions $u^{\Delta}$ generated by the u-scheme (4.6), (4.7), (4.9), (4.10) converge in the strong topology of $L^{1}\left(\Pi_{T}\right)$ to the unique weak solution of (1.1), (1.2) (in the sense of Definition 2.1).

Proof. Since $u^{\Delta} \in L^{\infty}\left(\Pi_{T}\right) \cap L^{\infty}(0, T ; B V(\mathbb{R})) \cap C^{1 / 2}\left(0, T ; L^{1}(\mathbb{R})\right)$, we deduce from (4.28) that there exists a sequence $\left\{\Delta_{i}\right\}_{i \in \mathbb{N}}$ with $\Delta_{i} \downarrow 0$ for $i \rightarrow \infty$ and a function $u \in L^{\infty}\left(\Pi_{T}\right) \cap L^{1}\left(\Pi_{T}\right) \cap L^{\infty}(0, T ; B V(\mathbb{R}))$ such that $u^{\Delta} \rightarrow u$ a.e. on $\Pi_{T}$. Moreover, in light of (4.29) we have $A\left(u^{\Delta}\right) \rightarrow A(u)$ strongly on $L_{\text {loc }}^{2}\left(\Pi_{T}\right)$, and we have that $A(u) \in L^{2}\left(0, T ; H^{1}(\mathbb{R})\right)$. Lemma 4.7 ensures that $u$ satisfies the initial condition (2.1). It remains to prove that $u$ satisfies the weak formulation (2.1). To this end, we apply a standard Lax-Wendroff-type argument. Now, multiplying (4.10) by $\int_{I_{j}} \varphi\left(x, t_{n}\right) \mathrm{d} x$, where $I_{j}:=\left[x_{j}, x_{j+1}\right]$ and $\varphi$ is a suitable smooth test function, and summing the results over $j \in \mathbb{Z}$, we obtain $W_{1}+W_{2}+W_{3}=0$, where we define

$$
\begin{aligned}
W_{1} & :=\sum_{n=0}^{N-1} \sum_{j \in \mathbb{Z}}\left(u_{j+1 / 2}^{n+1}-u_{j+1 / 2}^{n}\right) \int_{I_{j}} \varphi\left(x, t_{n}\right) \mathrm{d} x, \\
W_{2} & :=\lambda \sum_{n=0}^{N-1} \sum_{j \in \mathbb{Z}} \Delta_{+} G_{j}^{n} \int_{I_{j}} \varphi\left(x, t_{n}\right) \mathrm{d} x, \\
W_{3} & :=-\mu \sum_{n=0}^{N-1} \sum_{j \in \mathbb{Z}} \Delta^{2} A\left(u_{j+1 / 2}^{n}\right) \int_{I_{j}} \varphi\left(x, t_{n}\right) \mathrm{d} x .
\end{aligned}
$$

By standard summation by parts and using that $\varphi$ has compact support, we get

$$
\begin{aligned}
W_{1} & =-\Delta t \sum_{n=0}^{N-1} \sum_{j \in \mathbb{Z}} u_{j+1 / 2}^{n+1} \int_{I_{j}} \frac{\varphi\left(x, t_{n+1}\right)-\varphi\left(x, t_{n}\right)}{\Delta t} \mathrm{~d} x \\
W_{2} & :=-\Delta t \sum_{n=0}^{N-1} \sum_{j \in \mathbb{Z}} G_{j+1}^{n} \int_{I_{j}} \frac{\varphi\left(x+\Delta x, t_{n}\right)-\varphi\left(x, t_{n}\right)}{\Delta x} \mathrm{~d} x \\
W_{3} & :=-\Delta t \sum_{n=0}^{N-1} \sum_{j \in \mathbb{Z}} A\left(u_{j+1 / 2}^{n}\right) \int_{I_{j}} \frac{\varphi\left(x+\Delta x, t_{n}\right)-2 \varphi\left(x, t_{n}\right)+\varphi\left(x-\Delta x, t_{n}\right)}{\Delta x^{2}} \mathrm{~d} x .
\end{aligned}
$$

A direct application of the convergence of $u^{\Delta}$ gives us the desired result for $W_{1}$ and $W_{3}$. It remains to analyze $W_{2}$. Since for each fixed $n \in\{0, \ldots, N-1\}$, the data $\left\{v_{j}^{n}\right\}_{j \in \mathbb{Z}}$ are monotone, there exists an index $k$ such that $v_{k}^{n}<v^{*} \leq v_{k+1}^{n}$. Thus, if $\mathcal{A}, \mathcal{B}$ and $\mathcal{C}$ are the sets defined in (4.19), we may write $W_{2}=W_{2, \mathcal{A}}+W_{2, \mathcal{B}}+W_{2, \mathcal{C}}$, where the subindex denotes the summation over $j$ from the sets $\mathcal{A}, \mathcal{B}$ and $\mathcal{C}$, respectively. For $j \in \mathcal{A}$ we have

$$
G_{j+1}^{n}=\frac{\Delta_{+} \Phi\left(v_{j}^{n}\right)}{\Delta x}=\frac{\Delta_{+} \Phi\left(v_{j}^{n}\right)}{\Delta_{+} v_{j}^{n}} u_{j+1 / 2}^{n} \quad \text { if } u_{j+1 / 2}^{n} \neq 0 \text { and } G_{j+1}^{n}=0 \text { otherwise }
$$


since $\Phi^{\prime}>0$ for the values of $v_{j}^{n}$ with $j \in \mathcal{A}$. For $j \in \mathcal{C}$ we have

$$
G_{j+1}^{n}=\frac{\Delta_{+} \Phi\left(v_{j}^{n}\right)}{\Delta_{+} v_{j}^{n}} u_{j+1 / 2}^{n}+\frac{\Delta^{2} \Phi\left(v_{j+1}^{n}\right)}{\Delta x} \text { if } u_{j+1 / 2}^{n} \neq 0 \text { and } G_{j+1}^{n}=0 \text { otherwise. }
$$

We can write

$$
\begin{aligned}
\Delta^{2} \Phi\left(v_{j+1}^{n}\right)= & \Phi^{\prime}\left(v_{j+1}^{n}\right) \Delta_{+} u_{j+1 / 2}^{n} \Delta x \\
& +\frac{1}{2}\left(\Phi^{\prime \prime}\left(\xi_{j+3 / 2}^{n}\right)\left(\Delta_{+} v_{j+1}^{n}\right)^{2}+\Phi^{\prime \prime}\left(\xi_{j+1 / 2}\right)\left(\Delta_{+} v_{j}^{n}\right)\right),
\end{aligned}
$$

where $\xi_{j+1 / 2}^{n} \in\left[v_{j}^{n}, v_{j+1}^{n}\right]$. Using Lemmas 4.3 and 4.6 we get

$$
W_{2, \mathcal{C}}=-\Delta t \sum_{n=0}^{N-1} \sum_{j \in \mathcal{C}} \frac{\Delta_{+} \Phi\left(v_{j}^{n}\right)}{\Delta_{+} v_{j}^{n}} u_{j+1 / 2}^{n} \int_{I_{j}} \frac{\varphi\left(x+\Delta x, t_{n}\right)-\varphi\left(x, t_{n}\right)}{\Delta x} \mathrm{~d} x+\mathcal{O}(\Delta x) .
$$

The case $j \in \mathcal{B}$ can be treated in the same way as $j \in \mathcal{C}$ using that $\Phi^{\prime}\left(v^{*}\right)=0$ and that $\mathcal{B}$ is a finite index set. Taking the limit $\Delta \downarrow 0$ we finally get the result.

\section{Numerical examples}

The examples presented here illustrate the qualitative behavior of weak solutions of the initial value problem (1.1), (1.2) and the convergence properties of the numerical scheme. For the first purpose, we select a relatively fine discretization and present the corresponding numerical solutions as three-dimensional sequences of profiles at selected times or contour plots that should almost be free of numerical artifacts, while the convergence properties of the scheme are demonstrated by error histories in some examples. For all numerical examples we specify $\Delta x$ and use $\mu=\Delta t / \Delta x^{2}=0.1$, i.e., $\Delta t=0.1 \Delta x^{2}$.

5.1. Example 1. In Example 1 we calculate the numerical solution of (1.1), (1.2) for $\Phi(v)=-(1-v)^{2}$ and the degenerating integrated diffusion coefficient (1.12) with $u_{\mathrm{c}}=10$ and $a_{0}=0.1$. The initial datum is given by

$$
u_{0}(x)=\left\{\begin{array}{llll}
5 & \text { for } 0.1 \leq x \leq 0.2, & 7 & \text { for } 0.8 \leq x \leq 0.9 \\
8 & \text { for } 0.6 \leq x \leq 0.7, & 0 & \text { otherwise }
\end{array}\right.
$$

Note that $C_{0}=2$ in Example 1, where $C_{0}$ is defined in (1.10), and $v^{*}=C_{0} / 2=1$, so that the function $\Phi$ corresponds to (1.11), where the constant of integration is -1 , and that $u_{0}$ is chosen such that (1.6) is satisfied. Moreover, in our case $\Phi^{\prime \prime}(v)=-2<0$, and $\Phi(0)=\Phi\left(C_{0}\right)=-1$. Nagai and Mimura [32] show that under these conditions, and for the integrated diffusion coefficient given by (1.19), the solution converges in time to a compactly supported, stationary travelling-wave solution, which represents the aggregated group of individuals and is defined by the time-independent version of (1.1).

In Figure 5.1 we show the numerical approximations for $v$ and $u$ for $0 \leq t \leq 0.5$ and for $\Delta x=0.001$. As predicted, for each fixed time the data $\left\{v_{j}^{n}\right\}$ are monotonically increasing, and the numerical solution for $u$ indeed displays the aggregation phenomenon, and terminates in a stationary profile, even though the assumptions on $A$ stated in [32] are not satisfied here. This supports the conjecture that a similar travelling wave analysis can also be conducted in the present strongly degenerate case, to which we will return in a separate paper. 

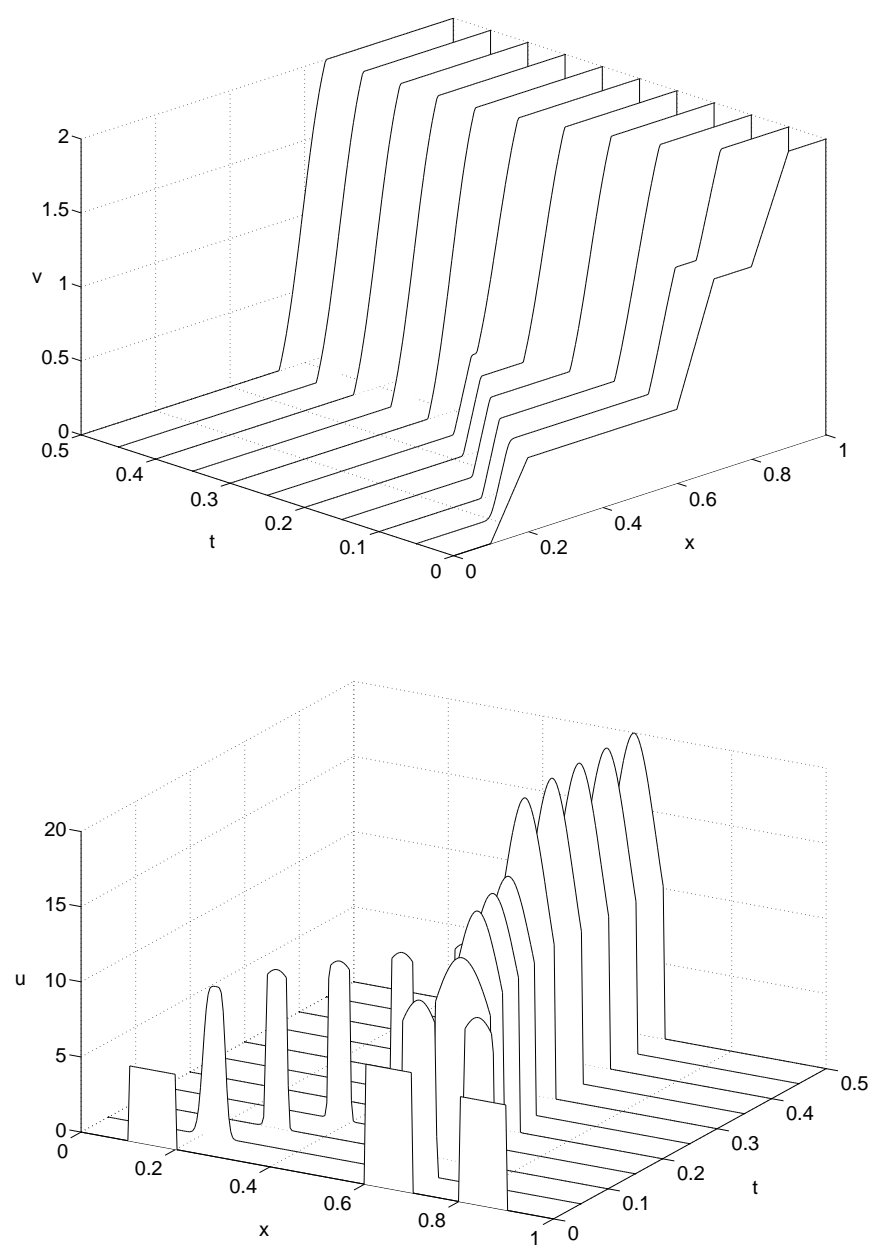

FIG. 5.1. Example 1: Numerical approximation of $v$ (top) and corresponding approximation of u (bottom), obtained via (4.2) with $\Delta x=0.001$.

In Table 5.1 we show the error at $t_{1}=0.1$ and $t_{2}=0.25$ in the $L^{1}$ norm for $u$ (denoted as $\left.e_{u}^{t_{i}}, i=1,2\right)$ and in the $L^{\infty}$ norm for $v$ (denoted as $e_{v}^{t_{i}}, i=1,2$ ), where we take as a reference the solution calculated with $\Delta x=0.0002$. We find an experimental rate of convergence in both cases greater than one. For small $\Delta x$ this behavior is possibly related to the proximity of the reference solution. One should expect a real order of convergence at most one since the $v$-scheme is monotone. Similar convergence rates have been observed for the other examples.

In Figure 5.2 we compare the numerical approximations for $v$ and $a$ for different mesh sizes at the simulated time $t=0.25$.

5.2. Example 2. This example represents a slight modification of Example 1, namely we choose $\Phi$ and $A$ as in Example 1, but we consider a smooth initial datum $u_{0}$ given by $u_{0}(x)=2-2 \cos (4 \pi x)$ for $x \in[0,1]$ and $u_{0}(x)=0$ otherwise. In Figure 5.3 

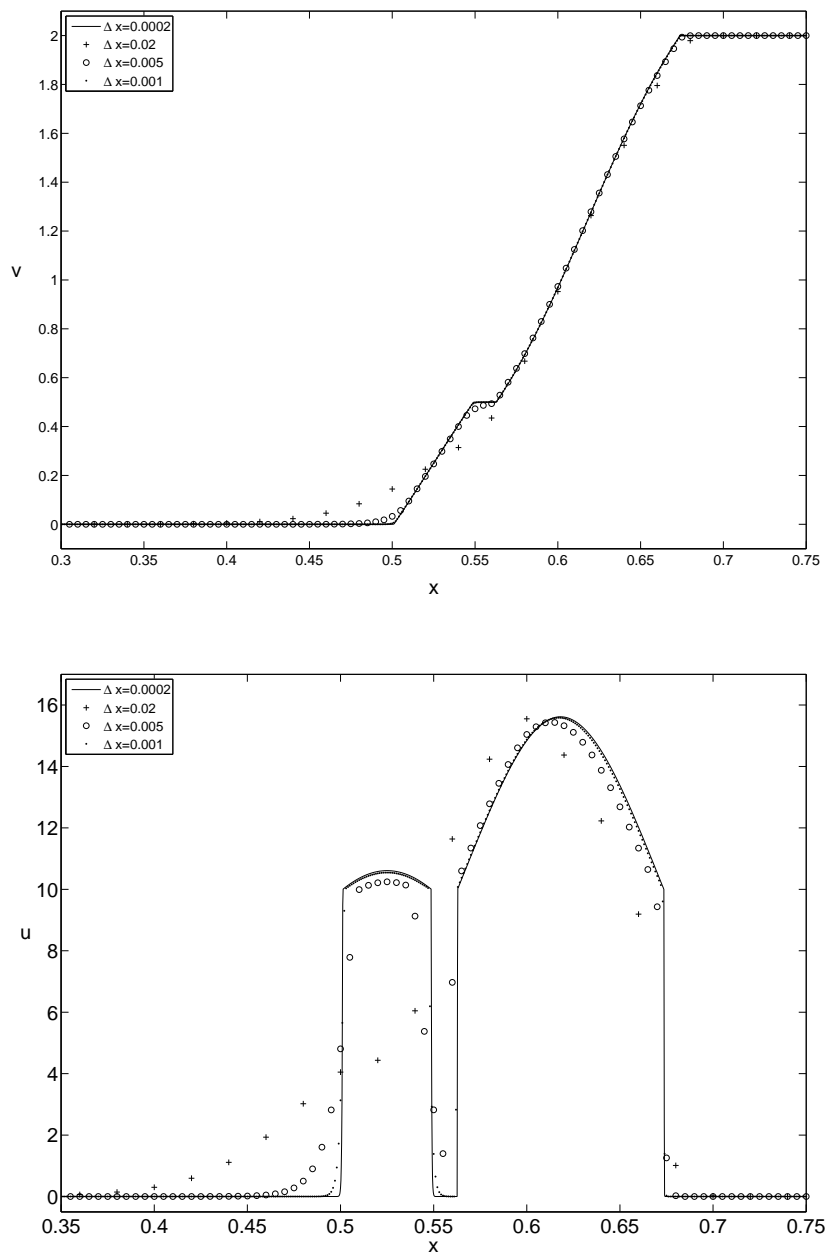

Fig. 5.2. Example 1: Numerical approximation of $v$ (top) and $u$ (bottom) for several mesh sizes at $t=0.25$.

we show the numerical approximation of $u$ for $0 \leq t \leq 0.5$ and $\Delta x=0.001$. We observe that strong discontinuities form after finite time from the smooth initial datum. This behavior contrasts with the regularity of other related problems [5, 24], where discontinuities can occur only if they are present in the initial data.

5.3. Example 3. We now choose $\Phi$ and $u_{0}$ as in Example 1, but define $A$ by

$$
A(u)= \begin{cases}0.05 u & \text { for } 0 \leq u \leq 5 \\ 0.25 & \text { for } 5<u \leq 10 \\ 0.05 u-0.25 & \text { for } u>10\end{cases}
$$

Figure 5.4 shows the results for $\Delta x=0.001$ and $t \in[0,0.5]$. Again, a stationary singlepeak solution is forming, including a jump between $u=5$ and $u=10$, in agreement with the flatness of $A(u)$ for $u \in[5,10]$. 


\begin{tabular}{ccccccccc}
\hline$\Delta x$ & $e_{v}^{t_{1}}$ & $\begin{array}{c}\text { conv. } \\
\text { rate }\end{array}$ & $e_{v}^{t_{2}}$ & $\begin{array}{c}\text { conv. } \\
\text { rate }\end{array}$ & $e_{u}^{t_{1}}$ & $\begin{array}{c}\text { conv. } \\
\text { rate }\end{array}$ & $e_{u}^{t_{2}}$ & $\begin{array}{c}\text { conv. } \\
\text { rate }\end{array}$ \\
\hline 0.020 & 0.239 & - & 0.317 & - & 0.915 & - & 0.695 & - \\
0.010 & 0.133 & 0.845 & 0.146 & 1.122 & 0.513 & 0.834 & 0.442 & 0.655 \\
0.005 & 0.061 & 1.135 & 0.069 & 1.070 & 0.246 & 1.062 & 0.200 & 1.144 \\
0.004 & 0.048 & 1.018 & 0.054 & 1.090 & 0.181 & 1.369 & 0.164 & 0.891 \\
0.002 & 0.021 & 1.168 & 0.024 & 1.161 & 0.082 & 1.150 & 0.073 & 1.163 \\
0.001 & 0.008 & 1.360 & 0.009 & 1.399 & 0.036 & 1.167 & 0.032 & 1.200 \\
\hline
\end{tabular}

TABLE 5.1. Example 1: Numerical error at $t_{1}=0.1$ and $t_{2}=0.25$.

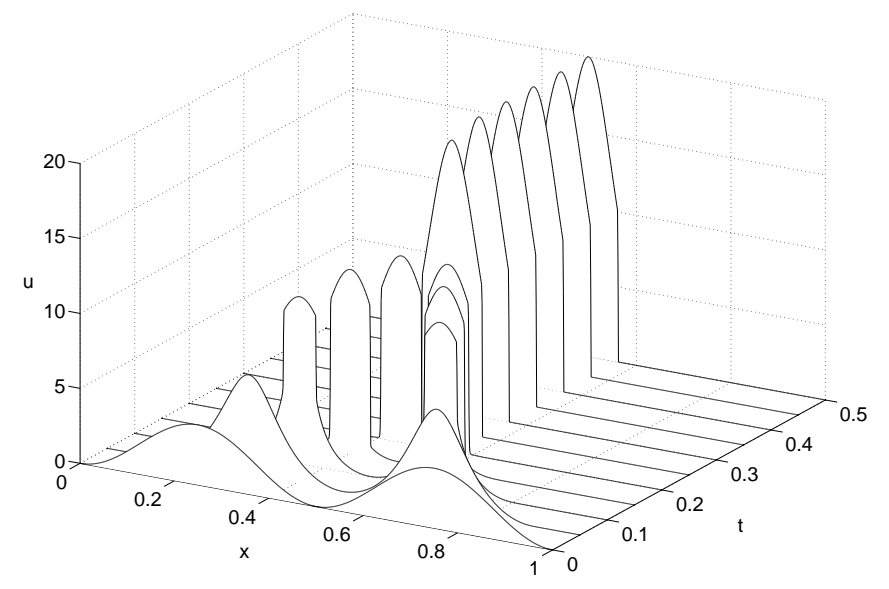

FIG. 5.3. Example 2: Numerical approximation of $u$, obtained via (4.2) with $\Delta x=0.001$.

5.4. Example 4. We now utilize the function $\Phi(v)=-0.5(\cos (v \pi)+1)$ combined with the degenerating integrated diffusion coefficient (1.12) with $u_{\mathrm{c}}=10$ and $a_{0}=0.1$ from Examples 1 and 2 and the initial datum

$$
u_{0}(x)=\left\{\begin{array}{llll}
10 & \text { for } x \in[0.05,0.15], & 9 & \text { for } x \in[0.6,0.7] \\
14 & \text { for } x \in[0.3,0.5], & 8 & \text { for } x \in[0.9,1] \\
& & 0 & \text { otherwise }
\end{array}\right.
$$

The result is shown in Figure 5.5 for $\Delta x=0.001$. We observe the formation of three groups, but the third moves to the right "looking for more" mass since it is not a full state, in the sense of the Nagai and Mimura [32] condition for the formation of stationary travelling waves. In addition to Figure 5.5 we show in Figure 5.6 a contour plot of the numerical approximation of $v$ for this example. The contour lines of $v$ correspond to trajectories of "individuals". This example has been included to illustrate the solution behaviour when $\Phi$ has several extrema and inflection points. 


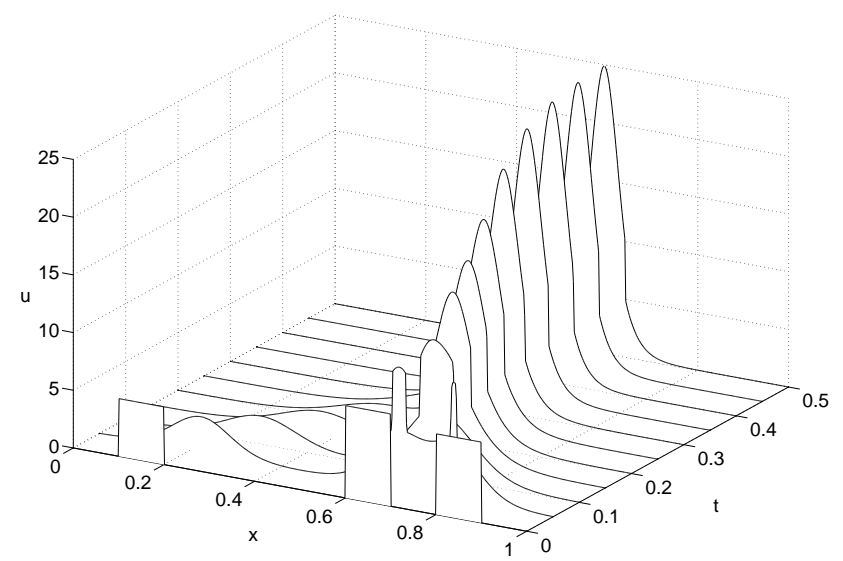

FIG. 5.4. Example 3: Numerical approximation of $u$, obtained via (4.2) for $\Delta x=0.001$.

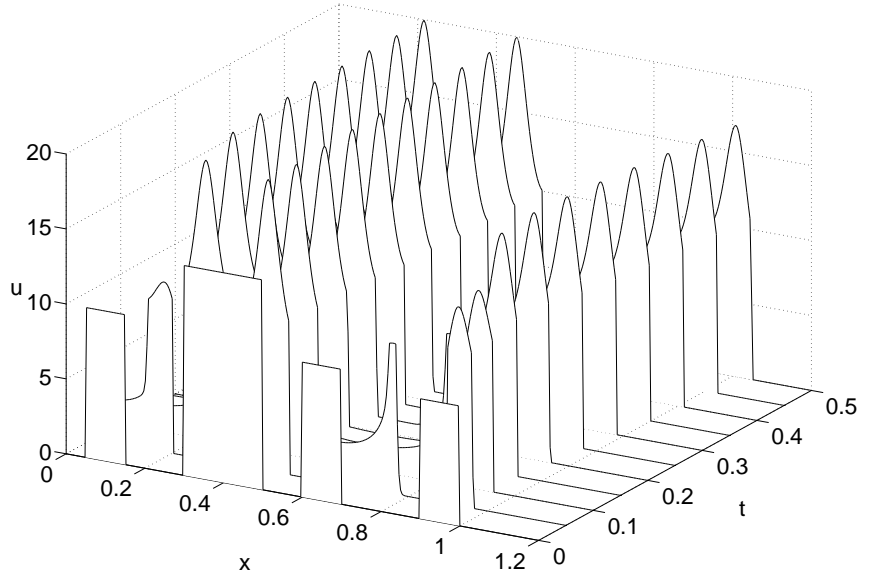

FIG. 5.5. Example 4: Numerical approximation of $u$, obtained via (4.2) for $\Delta x=0.001$.

5.5. Example 5. Here we calculate the numerical approximation of $u$ for $A$ as in Examples 1, 2 and 4, but with $\Phi$ and $u_{0}$ given by the respective equations

$$
\begin{aligned}
& \Phi(v)= \begin{cases}-0.5(\cos (v \pi)+1) & \text { for } 0 \leq v \leq 2, \\
(v-2)^{2}-1 & \text { for } v>2,\end{cases} \\
& u_{0}(x)=\left\{\begin{array}{llll}
14 & \text { for } x \in[0.15,0.3], & 18 & \text { for } x \in[0.8,0.95], \\
17 & \text { for } x \in[0.6,0.7], & 0 & \text { otherwise }
\end{array}\right.
\end{aligned}
$$

In Figure 5.7 we show the result for $\Delta x=0.001$. We see that the spare mass (i.e. the mass that can not get in the first group) "dilutes" to the right. This dissipation 


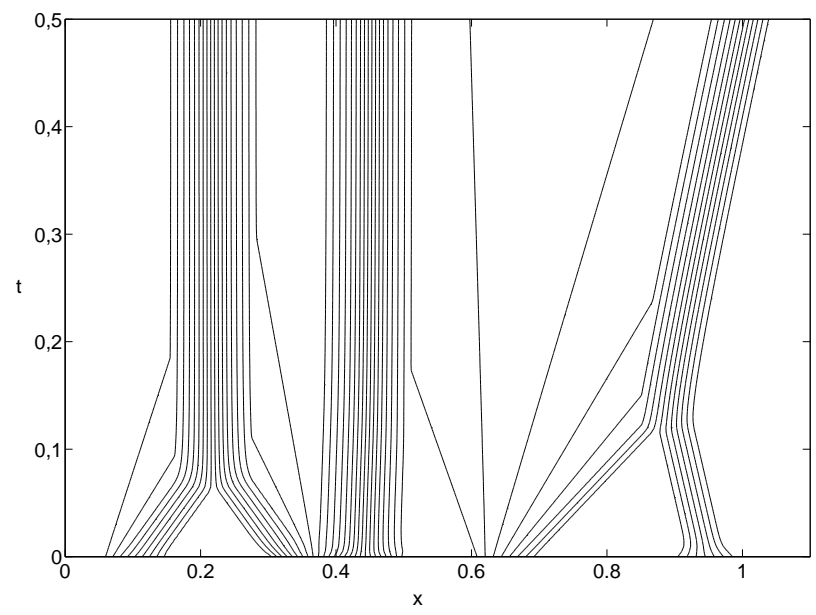

FIG. 5.6. Example 4: Contour lines of the numerical approximation of $v$ for $\Delta x=0.001$.

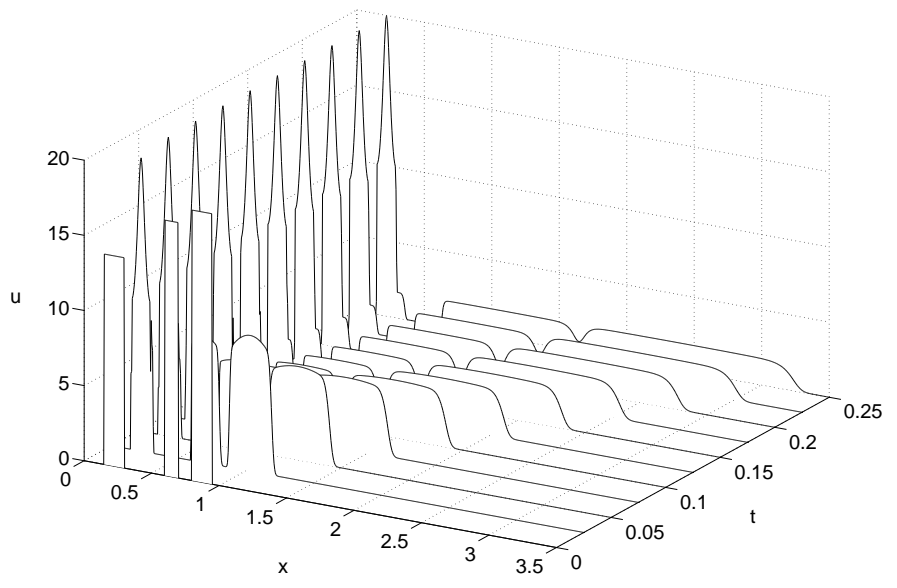

FiG. 5.7. Example 5: Numerical approximation of $u$, obtained via (4.2) for $\Delta x=0.001$.

of the right-moving mass is driven by the choice of $\Phi$, and not by that of $A$. Clearly, as in the previous example, $\Phi$ does not satisfy the assumption stated in (1.7). This example illustrates that solutions of (1.1), (1.2) will not always evolve into a finite number of stationary or moving, aggregated "herds".

Acknowledgements. FB acknowledges support by CONICYT fellowship. RB acknowledges support by Fondecyt project 1090456, Fondap in Applied Mathematics, project 15000001, and BASAL project CMM, Universidad de Chile and Centro de Investigación en Ingeniería Matemática $\left(\mathrm{CI}^{2} \mathrm{MA}\right)$, Universidad de Concepción. We thank the anonymous referees for a number of comments which helped to improve this paper. 


\section{REFERENCES}

[1] W. Alt, Degenerate diffusion equations with drift functionals modelling aggregation, Nonlin. Anal. TMA, 9, 811-836, 1985.

[2] J. Bedrossian, N. Rodríguez, and A. Bertozzi, Local and global well-posedness for aggregation equations and Patlak-Keller-Segel models with degenerate diffusion, preprint, Nonlinearity, submitted.

[3] A.L. Bertozzi and J. Brandman, Finite-time blow-up of $L^{\infty}$-weak solutions of an aggregation equation, Commun. Math. Sci., 8, 45-65, 2010.

[4] A.L. Bertozzi, J.A. Carrillo, and T. Laurent, Blow-up in multidimensional aggregation equations with mildly singular interaction kernels, Nonlinearity, 22, 683-710, 2009.

[5] A.L. Bertozzi and T. Laurent, Finite-time blow-up of solutions of an aggregation equation in $\mathbb{R}^{n}$, Commun. Math. Phys., 274, 717-735, 2007.

[6] A.L. Bertozzi, T. Laurent, and J. Rosado, $L^{p}$ theory for the multidimensional aggregation equation, Commun. Pure Appl. Math., 64, 45-83, 2011.

[7] A.L. Bertozzi and D. Slepčev, Existence and uniqueness of solutions to an aggregation equation with degenerate diffusion, Commun. Pure Appl. Anal., 9, 1617-1637, 2010.

[8] A. Blanchet, J.A. Carrillo, and P. Laurençot, Critical mass for a Patlak-Keller-Segel model with degenerate diffusion in higher dimensions, Calc. Var., 35, 133-168, 2009.

[9] M. Bodnar and J.J.L. Velazquez, An integro-differential equation arising as a limit of individual cell-based models, J. Diff. Eqs., 222, 341-380, 2006.

[10] H. Brézis and M.G. Crandall, Uniqueness of solutions of the initial-value problem for $u_{t}-$ $\Delta \varphi(u)=0$, J. Math. Pures et Appl., 58, 153-163, 1979.

[11] R. Bürger and K.H. Karlsen, On a diffusively corrected kinematic-wave traffic model with changing road surface conditions, Math. Models Meth. Appl. Sci., 13, 1767-1799, 2003.

[12] M. Burger, V. Capasso, and D. Morale, On an aggregation model with long and short range interactions, Nonlin. Anal. Real World Appl., 8, 939-958, 2007.

[13] J. Carrillo, Entropy solutions for nonlinear degenerate problems, Arch. Rational Mech. Anal., 147, 269-361, 1999.

[14] J.A. Carrillo, M. Di Francesco, A. Figalli, T. Laurent, and D. Slepčev, Global-in-time weak measure solutions and finite-time aggregation for nonlocal interaction equations, Duke Math. J., to appear.

[15] G.-Q. Chen and K.H. Karlsen, Quasilinear anisotropic degenerate parabolic equations with time-space dependent diffusion coefficients, Commun. Pure Appl. Anal., 4, 241-266, 2005.

[16] J.I. Diaz, T. Nagai, and S.I. Shmarev, On the interfaces in a nonlocal quasilinear degenerate equation arising in population dynamics, Japan J. Indust. Appl. Math., 13, 385-415, 1996.

[17] B. Engquist and S. Osher, One-sided difference approximations for nonlinear conservation laws, Math. Compt., 36, 321-351, 1981.

[18] S. Evje and K.H. Karlsen, Monotone difference approximations of BV solutions to degenerate convection-diffusion equations, SIAM J. Numer. Anal., 37, 1838-1860, 2000.

[19] S. Evje and K.H. Karlsen, Discrete approximations of BV solutions to doubly degenerate parabolic equations, Numer. Math., 86, 377-417, 2000.

[20] R. Eymard, T. Gallouët, R. Herbin, and A. Michel, Convergence of a finite volume scheme for nonlinear degenerate parabolic equations, Numer. Math., 92, 41-82, 2002.

[21] K.H. Karlsen and N.H. Risebro, Convergence of finite difference schemes for viscous and inviscid conservation laws with rough coefficients, M2AN Math. Model. Numer. Anal., 35, 239-269, 2001.

[22] K.H. Karlsen and N.H. Risebro, On the uniqueness and stability of entropy solutions for nonlinear degenerate parabolic equations with rough coefficients, Discr. Contin. Dyn. Syst., 9, 1081-1104, 2003.

[23] K. Kobayasi, The equivalence of weak solutions and entropy solutions of nonlinear degenerate second-order equations, J. Diff. Eqs., 189, 383-395, 2003.

[24] T. Laurent, Local and global existence for an aggregation equation, Commun. Part. Diff. Eqs., 32, 1941-1964, 2007.

[25] D. Li and J. Rodrigo, Finite-time singularities of an aggregation equation in $\mathbb{R}^{n}$ with fractional dissipation, Commun. Math. Phys., 287, 687-703, 2009.

[26] D. Li and J. Rodrigo, Refined blowup criteria and nonsymmetric blowup of an aggregation equation, Adv. in Math., 220, 1717-1738, 2009.

[27] D. Li and X. Zhang, On a nonlocal aggregation model with nonlinear diffusion, Discr. Cont. Dyn. Syst., 27, 301-323, 2010.

[28] A. Mogilner, L. Edelstein-Keshet, L. Bent, and A. Spiros, Mutual interactions, potentials, and individual distance in a social aggregation, J. Math. Biol., 47, 353-389, 2003. 
[29] D. Morale, V. Capasso, and K. Oelschläger, An interacting particle system modelling aggregation behavior: From individuals to populations, J. Math. Biol., 50, 49-66, 2005.

[30] T. Nagai, Some nonlinear degenerate diffusion equations with a nonlocally convective term in ecology, Hiroshima Math. J., 13, 165-202, 1983.

[31] T. Nagai and M. Mimura, Some nonlinear degenerate diffusion equations related to population dynamics, J. Math. Soc. Japan, 35, 539-562, 1983.

[32] T. Nagai and M. Mimura, Asymptotic behavior for a nonlinear degenerate diffusion equation in population dynamics, SIAM J. Appl. Math., 43, 449-464, 1983.

[33] T. Nagai and M. Mimura, Asymptotic behavior of the interfaces to a nonlinear degenerate diffusion equation in population dynamics, Japan J. Appl. Math., 3, 129-161, 1986.

[34] E. Rouvre and G. Gagneux, Solution forte entropique de lois scalaires hyperboliquesparaboliques dégénérées, C. R. Acad. Sci. Paris Sér. I, 329, 599-602, 1999.

[35] C.M. Topaz, A.L. Bertozzi and M.A. Lewis, A nonlocal continuum model for biological aggregation, Bull. Math. Biol., 68, 1601-1623, 2006. 